\title{
From little string free energies towards modular graph functions
}

\author{
Stefan Hohenegger \\ Univ Lyon, Univ Claude Bernard Lyon 1, CNRS/IN2P3, IP2I Lyon, UMR 5822, \\ F-69622, Villeurbanne, France \\ E-mail: s.hohenegger@ipnl.in2p3.fr
}

ABSTRACT: We study the structure of the non-perturbative free energy of a one-parameter class of little string theories (LSTs) of A-type in the so-called unrefined limit. These theories are engineered by $N$ M5-branes probing a transverse flat space. By analysing a number of examples, we observe a pattern which suggests to write the free energy in a fashion that resembles a decomposition into higher-point functions which can be presented in a graphical way reminiscent of sums of (effective) Feynman diagrams: to leading order in the instanton parameter of the LST, the $N$ external states are given either by the fundamental building blocks of the theory with $N=1$, or the function that governs the counting of BPS states of a single M5-brane coupling to one M2-brane on either side. These states are connected via an effective coupling function which encodes the details of the gauge algebra of the LST and which in its simplest (non-trivial) form is captured by the scalar Greens function on the torus. More complicated incarnations of this function show certain similarities with so-called modular graph functions, which have appeared in the study of Feynman amplitudes in string- and field theory. Finally, similar structures continue to exist at higher instanton orders, which, however, also contain contributions that can be understood as the action of (Hecke) operators on the leading instanton result.

Keywords: Field Theories in Higher Dimensions, String Duality, M-Theory, Solitons Monopoles and Instantons

ARXIV EPRINT: 1911.08172 


\section{Contents}

1 Introduction 1

2 Review: free energy 4

2.1 Little string free energy 4

2.2 Free energy for $N=1$

3 Example $N=\mathbf{2} \quad 6$

3.1 Order $Q_{R}^{1} \quad 6$

3.2 Order $Q_{R}^{2} \quad 8$

3.3 Order $Q_{R}^{3} \quad 11$

3.4 Nekrasov-Shatashvili limit 12

4 Example $N=3 \quad 14$

5 General structure of the unrefined free energy 16

$\begin{array}{lll}5.1 & \text { Summary of examples and general conjecture } & 16\end{array}$

$\begin{array}{ll}5.2 \text { Modular graph functions } & 18\end{array}$

$\begin{array}{lll}5.2 .1 & \text { Propagator } & 18\end{array}$

$\begin{array}{lll}\text { 5.2.2 } & \text { Modular graph functions } & 19\end{array}$

6 Conclusions 20

$\begin{array}{ll}\text { A Modular toolbox } & 21\end{array}$

$\begin{array}{ll}\text { A.1 Jacobi forms and Eisenstein series } & 21\end{array}$

A.2 Torus propagator 23

B Generating functions of multiple divisor sums 23

\section{Introduction}

In a series of works [1-4] it has been argued that a certain class of $\mathcal{N}=1$ supersymmetric little string theories (LSTs) [5-15] of A-type [16, 17] exhibits an intricate web of dualities. These theories are engineered in M-theory by $N$ parallel M5-branes spread out on a circle $S^{1}$ and probing a transverse $\mathbb{Z}_{M}$ geometry. A more geometric description of these theories can be given in terms of F-theory compactified on a particular type of toric Calabi-Yau threefold $X_{N, M}$ [18] which exhibits the structure of a double elliptic fibration.

While these LSTs contain string-like degrees of freedom (albeit without gravity), their low energy limits are supersymmetric gauge theories in six dimensions. It was argued in [3] 
that, for given $(N, M)$, the Calabi-Yau geometry $X_{N, M}$ engineers three (generically) different supersymmetric gauge theories, a fact which was termed triality. These theories are very different regarding their matter- and gauge content and are related through intrinsically non-perturbative duality maps, which mixes coupling constants, Coulomb branchand gauge parameters.

Furthermore, it was conjectured in [1] (and shown explicitly in a large number of examples) that the Calabi-Yau threefolds $X_{N, M}$ and $X_{N^{\prime}, M^{\prime}}$ are dual ${ }^{1}$ to one another if $N M=N^{\prime} M^{\prime}$ and $\operatorname{gcd}(N, M)=\operatorname{gcd}\left(N^{\prime}, M^{\prime}\right)$. It is therefore expected that the (nonperturbative) BPS-partition functions $\mathcal{Z}_{N, M}\left(\omega, \epsilon_{1,2}\right)$ and $\mathcal{Z}_{N^{\prime}, M^{\prime}}\left(\omega^{\prime}, \epsilon_{1,2}\right)$ agree once a suitable duality map (conjectured in [1] for generic $N$ and $M$ ) for the Kähler parameters (mapping $\omega \rightarrow \omega^{\prime}$ ) is taken into account. This equality was shown explicitly in [2] for $M=1$ and for general $(N, M)$ in [19] assuming a certain limit of two deformation parameters $\epsilon_{1,2}$ that are required to render $\mathcal{Z}_{N, M}\left(\omega, \epsilon_{1,2}\right)$ well defined. Combined with the triality of supersymmetric gauge theories argued in [3], this lead to the conjecture [4] of a vast web of dual gauge theories with gauge groups $\left[\mathrm{U}\left(N^{\prime}\right)\right]^{M^{\prime}}$ with $N M=N^{\prime} M^{\prime}$ and $\operatorname{gcd}(N, M)=\operatorname{gcd}\left(N^{\prime}, M^{\prime}\right)$.

While the dualities described above link very different looking theories in a nonperturbative fashion, they also imply symmetries for individual theories: focusing on $M=1$, it was first remarked in [20] that this web of dualities implies invariance of the partition function $\mathcal{Z}_{N, M}(\omega)$ under the group $\widetilde{\mathbb{G}}(N) \cong \mathbb{G}(N) \times \mathcal{S}_{N}$. Here $\mathcal{S}_{N} \cong \operatorname{Dih}_{N} \subset S_{N}$ is (a subgroup of) the Weyl group of the largest gauge group that can be engineered in a gauge theory from $X_{N, 1}$. Furthermore

$$
\mathbb{G}(N) \cong\left\{\begin{array}{l}
\operatorname{Dih}_{3} \text { if } N=1,3, \\
\operatorname{Dih}_{2} \text { if } N=2, \\
\operatorname{Dih}_{\infty} \text { if } N \geq 4,
\end{array}\right.
$$

where $\mathrm{Dih}_{\infty}$ is the group that is freely generated by two elements of order 2 .

Using this insight, the properties of the partition function $\mathcal{Z}_{N, 1}$ (or more specifically, the free energy $F_{N, 1}$ associated with it) have been re-examined in [21, 22]: among other things, on the one hand side it was argued in [21] that a particular subsector (called the reduced free energy) of $F_{N, 1}$ is invariant with respect to the paramodular group $\Sigma_{N}$, which in the Nekrasov-Shatashvili limit $[23,24] \epsilon_{2} \rightarrow 0$ is extended to $\Sigma_{N}^{*} \subset \operatorname{Sp}(4, \mathbb{Q})$. This result agrees with the earlier observation in [25] that the states contributing to the reduced free energy form a symmetric orbifold CFT and that an expansion of the former shows a very characteristic Hecke structure. On the other hand, by examining the examples $N=2,3,4$ up to order 3 in the instanton parameter $Q_{R}$ (from the point of view of the largest gauge group $\mathrm{U}(N)$ that can be engineered from $\left.X_{N, 1}\right)$ it was discussed in [22] that the remaining contributions to the free energy exhibit very suggestive structures. In particular, it was argued, that these BPS counting functions can be written using particular combinations of generating functions $T\left(\widehat{a}_{1}, \ldots, \widehat{a}_{N-1} ; \rho\right)$ of multiple divisor sums, which were introduced

\footnotetext{
${ }^{1}$ The duality $X_{N, M} \sim X_{N^{\prime}, M^{\prime}}$ means that these two manifolds share the same extended Kähler moduli space, i.e. their Kähler moduli spaces are connected through so-called flop transformations. We refer to [1] for details.
} 
in [26]. These functions are a generalisation of quasi-Jacobi forms and combine in a natural fashion the modular properties (acting on the modular parameter $\rho$ ) of the free energy with the $\widehat{\mathfrak{a}}_{N+1}$ (affine) gauge algebra (with roots $\widehat{a}_{1, \ldots, N-1}$ ).

As was already remarked in [26], the generating functions $T\left(\widehat{a}_{1}, \ldots, \widehat{a}_{N-1} ; \rho\right)$ are also intimately connected to multiple zeta values and can be expanded in terms of (reduced) polylogarithms. The latter have in recent years attracted a lot of attention in the study of (loop) amplitudes in (supersymmetric) string theories (see e.g. [27-38] and references therein for an overview). The current paper is motivated by extending this connection and extracting a class of functions from the free energy $F_{N, 1}$, which show a certain resemblance of so-called (modular) graph functions (see [32, 33, 38-45]) that have been studied recently in the literature. To this end, we consider the so-called unrefined limit $\epsilon_{1}=-\epsilon_{2}=\epsilon$ and study instanton expansions of the free energy for $N=2,3$ up to order $Q_{R}^{3}$ :

$$
F_{N, 1}\left(\widehat{a}_{1}, \ldots, \widehat{a}_{N-1}, \rho, R, S, \epsilon\right)=\sum_{r=1}^{\infty} Q_{R}^{r} \sum_{s=0}^{\infty} \epsilon^{2 s-2} B_{(s)}^{(N, r)}\left(\widehat{a}_{1}, \ldots, \widehat{a}_{N-1}, \rho, S\right) .
$$

The latter exhibit a number of very interesting patterns, which can schematically be represented in the form of graphs, that resemble effective higher point functions (even similar to Feynman diagrams): indeed, to leading instanton order (i.e. for $r=1$ in (1.2)) we find that we can write

$$
B_{(s)}^{(N, r=1)}=H_{(s)}^{(0,1)}(\rho, S) \sum_{i=0}^{N-1}\left(W_{(0)}(\rho, S)\right)^{N-1-i}\left(H_{(0)}^{(0,1)}(\rho, S)\right)^{i} \mathcal{O}^{(N), i}\left(\widehat{a}_{1, \ldots, N-1}, \rho\right)
$$

which we have verified for $N=2,3$ up to order $s=4$ and also partially for $N=4$, using the data for $s=0$ provided in [22]. In (1.3) we have as the fundamental building blocks $H_{(s)}^{(0,1)}=B_{(s)}^{(N=1, r=1)}$ and $W_{(0)}$, which is a particular quasi-Jacobi form of weight 0 and index 1 (see (3.3) for the precise definition). The latter are 'coupled' through the coupling function $\mathcal{O}^{(N), i}$, which is independent of $S$ and $s$ and depends on the roots of the gauge algebra $\widehat{\mathfrak{a}}_{N+1}$. The form (1.3) can graphically be presented as an $N$-point function, where the external legs are given by $H_{(s)}^{(0,1)}$ and $W_{(0)}$ respectively, which are connected via $\mathcal{O}^{(N), i}$ (see figure 9). While a priori nothing more than an amusing graphical representation, this interpretation seems to go beyond a mere mnemonic device: for the examples $N=2,3,4$ we find that $\mathcal{O}^{(N), 0}=N$, while $\mathcal{O}^{(N), 1}$ is in fact (up to a $\widehat{a}_{1, \ldots, N-1 \text {-independent term) }}$ related to the scalar Greens function on the torus (see appendix A.2). Moreover, the real part of $\mathcal{O}^{(N), 1}$ is the fundamental building block for the study of graph functions in [33]. Higher $\mathcal{O}^{(N), i}$, for $i>1$, are more involved. However, using the presentation in terms of the $T\left(\widehat{a}_{1}, \ldots, \widehat{a}_{N-1} ; \rho\right)$ mentioned above, they can be written as combinations of polylogarithms - a property they share in common with the graph functions in [33]. We leave it to further work to analyse these functions in detail.

This paper is organised as follows: in section 2 we review the instanton partition function $\mathcal{Z}_{N, 1}\left(\omega, \epsilon_{1,2}\right)$ and free energy $F_{N, 1}\left(\omega, \epsilon_{1,2}\right)$ as well as their symmetries found in [21, 22]. In sections 3 and 4 we study the cases $N=2$ and $N=3$ respectively, which reveal the patterns mentioned above. In section 5 we summarise these examples and formulate a 
conjecture for the general structure in the form of (1.2). We also discuss similarities of the free energy with modular graph functions in the literature of scattering amplitudes in string- and field theory. Section 6 contains our conclusions and an outlook for future work. Furthermore, two appendices contain a short review on modular objects and the generating functions of multiple divisor sums.

\section{Review: free energy}

\subsection{Little string free energy}

In order to set the stage for subsequent sections, we start by reviewing the partition function and free energy of a class of little string theories of A-type $[1,2,16,17]$. The latter can be engineered through F-theory compactified on a class of toric, non-compact Calabi-Yau threefolds [18], which are called $X_{N, M}$ for $N, M \in \mathbb{N}$. The corresponding non-perturbative BPS partition function $\mathcal{Z}_{N, M}\left(\omega, \epsilon_{1,2}\right)$ of these LSTs is captured by the topological string partition function on $X_{N, M}$ [16] (see also [46-49]), which can be computed in a very efficient manner using the refined topological vertex (see [50-52]): indeed, in [2] a general building block $W_{\beta_{1}, \ldots, \beta_{N}}^{\alpha_{1}, \ldots, \alpha_{N}}\left(\widehat{a}_{1, \ldots, N}, S ; \epsilon_{1,2}\right)$ was computed that is labelled by $2 N$ sets of integer partitions $\alpha_{1, \ldots, N}$ and $\beta_{1, \ldots, N}$ and that depends on (a subset of) the Kähler parameters of $X_{N, M}$. The latter allows to compute the full instanton partition function by 'gluing together' $M$-copies of itself, weighted in a suitable manner by the instanton parameters. In the case $M=1$, only a single building block is necessary and the non-perturbative part of the partition function can be written as

$$
\mathcal{Z}_{N, 1}\left(\widehat{a}_{1, \ldots, N-1}, \rho, S, R ; \epsilon_{1,2}\right)=\sum_{\{\alpha\}}\left(\prod_{i=1}^{N} Q_{m_{i}}^{\left|\alpha_{i}\right|}\right) W_{\alpha_{1}, \ldots, \alpha_{N}}^{\alpha_{1}, \ldots, \alpha_{N}}\left(\widehat{a}_{1, \ldots, N-1}, \rho, S ; \epsilon_{1,2}\right) .
$$

Concerning the Kähler parameters of $X_{N, 1}$, we use the same basis that was introduced in [3], i.e. $\left(\widehat{a}_{1}, \ldots, \widehat{a}_{N-1}, \rho, S, R\right)$, where the $\widehat{a}_{1, \ldots, N-1}$ and $\rho$ play the roles of roots of the affine $\widehat{\mathfrak{a}}_{N+1}$ gauge algebra, $S$ plays the role of a mass parameter, while $R$ is related to the coupling constant. The $Q_{m_{i}}=e^{2 \pi i m_{i}}$ with $m_{i}=m_{i}\left(R, S, \widehat{a}_{1, \ldots, N-1}, \rho\right)$ appearing in (2.1) are linear combinations of $\left(\widehat{a}_{1, \ldots, N-1}, S, R\right)$ and contain the only dependence on $R$. Thus, $(2.1)$ is essentially an expansion in powers of $Q_{R}=e^{2 \pi i R}$, which can therefore be identified with an instanton expansion.

Rather than working with the partition function $\mathcal{Z}_{N, 1}$, we work with the free energy

$$
F_{N, 1}\left(\widehat{a}_{1, \ldots, N-1}, \rho, S, R ; \epsilon_{1,2}\right)=\operatorname{PLog} \mathcal{Z}_{N, 1}\left(\widehat{a}_{1, \ldots, N-1}, \rho, S, R ; \epsilon_{1,2}\right),
$$

which affords the following Fourier expansion

$$
F_{N, 1}\left(\widehat{a}_{1}, \ldots, \widehat{a}_{N}, S, R ; \epsilon_{1,2}\right)=\sum_{s_{1}, s_{2}=0}^{\infty} \sum_{r=0}^{\infty} \sum_{i_{1}, \ldots, i_{N}}^{\infty} \sum_{k \in \mathbb{Z}} \epsilon_{1}^{s_{1}-1} \epsilon_{2}^{s_{2}-1} f_{i_{1}, \ldots, i_{N}, k, r}^{\left(s_{1}, s_{2}\right)} Q_{\widehat{a}_{1}}^{i_{1}} \ldots Q_{\widehat{a}_{N}}^{i_{N}} Q_{S}^{k} Q_{R}^{r}
$$


with the coefficients $f_{i_{1}, \ldots, i_{N}, k, r}^{\left(s_{1}, s_{2}\right)}{ }^{2}$ We use the same notation as in [21, 22], i.e. $\rho=\sum_{i=1}^{N} \widehat{a}_{i}$ and

$$
Q_{\widehat{a}_{i}}=e^{2 \pi i \widehat{a}_{i}}, \quad Q_{\rho}=e^{2 \pi i \rho}=\prod_{i=1}^{N} Q_{\widehat{a}_{i}}, \quad Q_{S}=e^{2 \pi i S}, \quad Q_{R}=e^{2 \pi i R}, \quad \text { for } \quad i=1, \ldots, N,
$$

and we introduce the following partial expansion

$$
H_{\left(s_{1}, s_{2}\right)}^{\left(i_{1}, \ldots, i_{N}, r\right)}(\rho, S)=\sum_{\ell=0}^{\infty} \sum_{k \in \mathbb{Z}} f_{i_{1}+\ell, i_{2}+\ell, \ldots, i_{N}+\ell, k, r}^{\left(s_{1}, s_{2}\right)} Q_{S}^{k} Q_{\rho}^{\ell}, \quad \forall i_{1, \ldots, N} \in \mathbb{N} \cup\{0\} .
$$

The free energy can be formally ${ }^{3}$ recovered as

$$
F_{N, 1}\left(\widehat{a}_{1}, \ldots, \widehat{a}_{N}, S, R ; \epsilon_{1,2}\right)=\sum_{s_{1}, s_{2}=0}^{\infty} \sum_{r=0}^{\infty} \epsilon_{1}^{s_{1}-1} \epsilon_{2}^{s_{2}-1} Q_{R}^{r} B_{\left(s_{1}, s_{2}\right)}^{(N, r)}\left(\widehat{a}_{1}, \ldots, \widehat{a}_{N-1}, \rho, S\right),
$$

with the functions

$$
B_{\left(s_{1}, s_{2}\right)}^{(N, r)}=\sum_{i_{1}, \ldots, i_{N}}^{\prime} H_{\left(s_{1}, s_{2}\right)}^{\left(i_{1}, \ldots, i_{N}, r\right)}(\rho, S) Q_{\widehat{a}_{1}}^{i_{1}} \ldots Q_{\widehat{a}_{N}}^{i_{N}},
$$

and the sum is understood to run over all $\left(i_{1}, \ldots, i_{N}\right)$ such that (at least) one $i_{a}=0$. Finally, we define the coefficients which appear in the expansion of $F_{N, 1}$ in the unrefined case $\epsilon_{1}=-\epsilon_{2}=\epsilon$

$$
H_{(s)}^{\left(i_{1}, \ldots, i_{N}, r\right)}=\sum_{s_{1}+s_{2}=2 s}(-1)^{s_{2}-1} H_{\left(s_{1}, s_{2}\right)}^{\left(i_{1}, \ldots, i_{N}, r\right)}, \quad B_{(s)}^{(N, r)}=\sum_{s_{1}+s_{2}=2 s}(-1)^{s_{2}-1} B_{\left(s_{1}, s_{2}\right)}^{(N, r)} .
$$

Numerous examples of $H_{\left(s_{1}, s_{2}\right)}^{\left(i_{1}, \ldots, i_{N}, r\right)}$ have been computed in [22] and its properties have been discussed in detail. In particular, it has been argued that a suitable set of functions to represent the $H_{\left(s_{1}, s_{2}\right)}^{\left(i_{1}, \ldots, i_{N}, r\right)}$ are the generating functions of multiple divisor sums $T\left(X_{1}, \ldots, X_{\ell} ; \rho\right)$ that have been first introduced in [26] and which are defined in (B.1). Rather than recounting all the properties of $B_{\left(s_{1}, s_{2}\right)}^{(N, r)}$ found in [22], we shall instead give as an example the simplest case (namely $N=1$ ) in the following, whose expansion will provide us with the general building blocks that are used in the remainder of this paper.

\section{$2.2 \quad$ Free energy for $N=1$}

The unrefined free energy for $N=1$ (with $\epsilon_{1}=-\epsilon_{2}=\epsilon$ ) is of the form

$$
\begin{aligned}
F_{1,1}\left(\rho, S, R ; \epsilon_{1}=-\epsilon_{2}=\epsilon\right) & =\sum_{s=0}^{\infty} \epsilon^{2 s-2} \sum_{r=0}^{\infty} Q_{R}^{r} H_{(s)}^{(0,1)}(\rho, S), \\
\text { with } H_{(s)}^{(0,1)} & =\sum_{s_{1}+s_{2}=2 s}(-1)^{s_{2}-1} H_{\left(s_{1}, s_{2}\right)}^{(0,1)} .
\end{aligned}
$$

\footnotetext{
${ }^{2}$ Due to the plethystic logarithm PLog,$F_{N, 1}$ only counts single particle BPS states. The former is defined as $\mathrm{PLog} \mathcal{Z}_{N, 1}\left(\widehat{a}_{1, \ldots, N-1}, \rho, S, R ; \epsilon_{1,2}\right)=\sum_{k=1}^{\infty} \frac{\mu(k)}{k} \ln \mathcal{Z}_{N, 1}\left(k \widehat{a}_{1, \ldots, N}, k S, k R ; k \epsilon_{1,2}\right)$, where $\mu$ is the Möbius function.

${ }^{3}$ Here we do not worry about convergence: we shall reformulate the free energy in (modular) objects, for which we utilise a suitable summation procedure [53] to guarantee convergence. See [22] for more details.
} 
To order $Q_{R}^{1}$ we have concretely

$$
\sum_{s=0}^{\infty} \epsilon^{2 s-2} H_{(s)}^{(0,1)}=-\frac{\phi_{-2,1}}{\epsilon^{2}}+\frac{\phi_{0,1}}{24}-\sum_{n=1}^{\infty} \epsilon^{2 n} \frac{(-1)^{n} B_{2 n+2} E_{2 n+2}(\rho) \phi_{-2,1}(\rho, S)}{(2 n-1) ! !(2 n+2) ! !},
$$

while for higher orders in $Q_{R}^{r}$ (with $r>1$ ) it was found in [25]

$$
H_{(s)}^{(0, k)}(\rho, S)=\mathcal{T}_{k}\left(H_{(s)}^{(0,1)}(\rho, S)\right)=\sum_{a \mid k} a^{w-1} \mu(a) \mathcal{H}_{k / a}\left(H_{(s)}^{(0,1)}(a \rho, a S)\right),
$$

where $\mathcal{H}_{k}$ is the $k$-th Hecke operator (see eq. (A.10)) and $\mu$ is the Möbius function. For later convenience, we explicitly tabulate the first few coefficient-functions up to order $Q_{R}^{2}$ as follows

\begin{tabular}{|c|r|r|}
\hline$s$ & $H_{(s)}^{(0,1)}$ & $H_{(s)}^{(0,2)}$ \\
\hline 0 & $-\phi_{-2,1}$ & $-\frac{\phi_{-2,1}}{24}\left(\phi_{0,1}+\psi_{2} \phi_{-2,1}\right)$ \\
\hline 1 & $\frac{\phi_{0,1}}{24}$ & $\frac{1}{576}\left[24 E_{4} \phi_{-2,1}^{2}+\left(\phi_{0,1}-2 \psi_{2} \phi_{2,1}\right)\left(\phi_{0,1}+3 \psi_{2} \phi_{-2,1}\right)\right]$ \\
\hline 2 & $-\frac{E_{4} \phi_{-2,1}}{240}$ & $\frac{\phi_{-2,1}}{11520}\left[5 \psi_{2}^{2}\left(\phi_{0,1}+4 \psi_{2} \phi_{-2,1}\right)-2 E_{4}\left(11 \phi_{0,1}+41 \psi_{2} \phi_{2,1}\right)\right]$ \\
\hline 3 & $-\frac{E_{6} \phi_{-2,1}}{6048}$ & $\frac{\phi_{-2,1}}{290304}\left[252 E_{4}^{2} \phi_{-2,1}+87 E_{4} \psi_{2} \phi_{0,1}-2\left(8 E_{6} \psi_{2} \phi_{-2,1}+\psi_{2}^{3}\left(11 \phi_{0,1}+7 \psi_{2} \phi_{-2,1}\right)\right)\right]$ \\
\hline 4 & $-\frac{E_{4}^{2} \phi_{-2,1}}{172800}$ & $\frac{\phi_{-2,1}}{74649600}\left[-2178 E_{4}^{2} \phi_{0,1}+9372 E_{4} E_{6} \phi_{-2,1}+\psi_{2}\left(240 E_{6} \phi_{0,1}-416 E_{6} \psi_{2} \phi_{-2,1}\right.\right.$ \\
& $\left.\left.+105 \psi_{2}^{3} \phi_{0,1}-242 \psi_{2}^{4} \phi_{-2,1}\right)\right]$ \\
\hline
\end{tabular}

The Eisenstein series $E_{2 k}$ and $\psi_{2}$ and the Jacobi forms $\phi_{-2,1}$ and $\phi_{0,1}$ are defined in appendix A.

\section{Example $N=2$}

We start by considering the free energy for $N=2$. More concretely, using the same notation as in $[21,22]$, we can separate the latter into two pieces (for $r \geq 1$ )

$$
B_{(s)}^{(2, r)}=H_{(s)}^{(0,0, r)}(\rho, S)+K_{(s)}^{(r, 2)}\left(\rho, S, \widehat{a}_{1}\right), \quad \text { with } \quad K_{(s)}^{(1,2)}=\sum_{n=1}^{\infty} H_{(s)}^{(n, 0, r)}\left(Q_{\widehat{a}_{1}}^{n}+\frac{Q_{\rho}^{n}}{Q_{\widehat{a}_{1}}^{n}}\right) .
$$

Below we shall analyse the orders $r=1,2,3$ in more detail.

\section{$3.1 \quad$ Order $Q_{R}^{1}$}

For low values of $s$ we find for the terms $H_{(s)}^{(n, 0,1)}$ in (3.1) the following expressions

$$
\begin{aligned}
& H_{(0)}^{(n, 0,1)}=-\frac{2 n}{1-Q_{\rho}^{n}} \phi_{-2,1}^{2}, \\
& H_{(1)}^{(n, 0,1)}=\frac{n}{12\left(1-Q_{\rho}^{n}\right)} \phi_{0,1} \phi_{-2,1}, \\
& H_{(2)}^{(n, 0,1)}=-\frac{n}{120\left(1-Q_{\rho}^{n}\right)} E_{4} \phi_{-2,1}^{2}, \\
& H_{(3)}^{(n, 0,1)}=-\frac{n}{3024\left(1-Q_{\rho}^{n}\right)} E_{6} \phi_{-2,1}^{2}, \\
& H_{(4)}^{(n, 0,1)}=-\frac{n}{86400\left(1-Q_{\rho}^{n}\right)} E_{4}^{2} \phi_{-2,1}^{2} .
\end{aligned}
$$




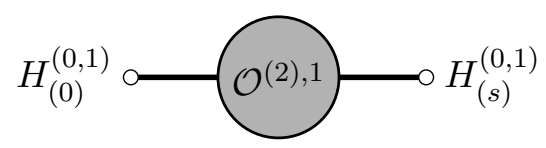

Figure 1. Coupling function $\mathcal{O}^{(2), 1}$ appearing in the $\epsilon$-expansion of $K_{(s)}^{(1,2)}$.

Comparing with the $H_{(s)}^{(0,1)}$ appearing in the expansion of the free energy of $N=1$ (as tabulated in section 2.2), these examples exhibit a pattern which suggests ${ }^{4}$

$$
K_{(s)}^{(1,2)}=H_{(0)}^{(0,1)} \mathcal{O}^{(2), 1} H_{(s)}^{(0,1)}, \quad \text { where } \quad \mathcal{O}^{(2), 1}\left(\widehat{a}_{1}, \rho\right)=-\sum_{n=1}^{\infty} \frac{2 n}{1-Q_{\rho}^{n}}\left(Q_{\widehat{a}_{1}}^{n}+\frac{Q_{\rho}^{n}}{Q_{\widehat{a}_{1}}^{n}}\right)
$$

This relation can be represented graphically as in figure 1 , where $H_{(0)}^{(0,1)}$ is coupled to $H_{(s)}^{(0,1)}$ through the coupling function $\mathcal{O}^{(2), 1}$. Notice that the latter is independent of $s$ and only depends on $\left(\widehat{a}_{1}, \rho\right)$, but not $S$ (whose dependence in figure 1 is only given through $H_{(s)}^{(0,1)}$ and $\left.H_{(0)}^{(0,1)}\right)$.

Similar to $K_{(s)}^{(1,2)}$, we can compute $H_{(s)}^{(0,0,1)}$, where the first few examples are

$$
\begin{aligned}
H_{(0)}^{(0,0,1)} & =-\frac{1}{12} \phi_{-2,1}\left(\phi_{0,1}+2 E_{2} \phi_{-2,1}\right), \\
H_{(1)}^{(0,0,1)} & =\frac{1}{288} \phi_{0,1}\left(\phi_{0,1}+2 E_{2} \phi_{-2,1}\right), \\
H_{(2)}^{(0,0,1)} & =-\frac{1}{2880} E_{4} \phi_{-2,1}\left(\phi_{0,1}+2 E_{2} \phi_{-2,1}\right), \\
H_{(3)}^{(0,0,1)} & =-\frac{1}{72576} E_{6} \phi_{-2,1}\left(\phi_{0,1}+2 E_{2} \phi_{-2,1}\right), \\
H_{(4)}^{(0,0,1)} & =-\frac{1}{2073600} E_{4}^{2} \phi_{-2,1}\left(\phi_{0,1}+2 E_{2} \phi_{-2,1}\right) .
\end{aligned}
$$

In the same manner as (3.2), these examples suggest the pattern

$$
H_{(s)}^{(0,0,1)}(\rho, S)=W_{(0)} \mathcal{O}^{(2), 0} H_{(s)}^{(0,1)}, \quad \text { where } \quad\left\{\begin{array}{l}
\mathcal{O}^{(2), 0}=2 \\
W_{(0)}=\frac{1}{24}\left(\phi_{0,1}+2 E_{2} \phi_{-2,1}\right) .
\end{array}\right.
$$

As above, this relation can be represented graphically as in figure 2 , where $W_{(0)}$ is coupled to $H_{(s)}^{(0,1)}$ through $\mathcal{O}^{(2), 0}$. The quasi-Jacobi form $W_{(0)}$ is the leading term in the expansion $W\left(\rho, S, \epsilon_{1,2}\right)=\frac{\theta_{1}\left(\rho ; S+\epsilon_{-}\right) \theta_{1}\left(\rho ; S-\epsilon_{-}\right)-\theta_{1}\left(\rho ; S+\epsilon_{+}\right) \theta_{1}\left(\rho ; S-\epsilon_{+}\right)}{\theta_{1}\left(\rho ; \epsilon_{1}\right) \theta_{1}\left(\rho ; \epsilon_{2}\right)}=W_{(0)}+\mathcal{O}\left(\epsilon_{1}, \epsilon_{2}\right)$,

which appeared in [16] (see also [21]), as the function governing the counting of BPS states of a (local) M5-brane with a single M2-brane ending on it on either side. We refer the reader to $[16,21]$ for more details.

\footnotetext{
${ }^{4}$ Our notation is explained in more detail in section 5: there we shall denote general coupling functions $\mathcal{O}^{(N), i}$, where $i$ is a summation index labelling different classes of couplings.
} 


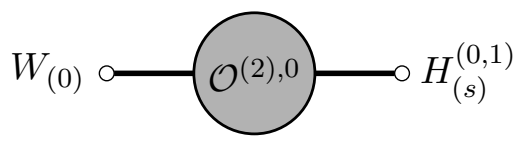

Figure 2. Coupling function $\mathcal{O}^{(2), 0}$ appearing in the expansion of the free energy $H_{(s)}^{(1,2)}$ in powers of $\epsilon$.

In (3.2) and (3.3) we have called $\mathcal{O}^{(2), 1}$ and $\mathcal{O}^{(2), 0}$ coupling functions and have graphically represented the contribution to the free energy in figure 1 and figure 2 like a(n effective) 'propagator' appearing in a two-point function. In the case of the former, this connection can be made more concrete: $\mathcal{O}^{(2), 1}$ can (up to a non-holomorphic contribution) be related to (the derivative of) the scalar two-point function on the torus, as we shall explain explicitly in section 5.2.1.

\section{$3.2 \quad$ Order $Q_{R}^{2}$}

We have seen that to leading instanton order, the free energy can be presented as a 'twopoint function' involving the effective coupling functions $\mathcal{O}^{(2), 0}$ and $\mathcal{O}^{(2), 1}$. Higher orders in $Q_{R}$ are more involved, however, still exhibit very interesting similar structures.

Indeed, slightly adopting the notation used in [22], for $r=2$ in (3.1), we can write

$$
H_{(s)}^{(n, 0,2)}=\sum_{i=1}^{4}\left[g_{(s)}^{i,(n, 2)}+\theta_{n, 2} h_{(s)}^{i,(n, 2)}\right] \frac{n \phi_{-2,1}^{5-i} \phi_{0,1}^{i-1}}{1-Q_{\rho}^{n}}, \quad \text { with } \quad \theta_{a, b}=\left\{\begin{array}{l}
0 \text { if } \operatorname{gcd}(a, b)=1 \\
1 \text { if } \operatorname{gcd}(a, b)>1
\end{array}\right.
$$

where we can tabulate the coefficients $g_{(s)}^{i,(n, 2)}$ as follows

\begin{tabular}{|l|r|r|r|r|}
\hline$s$ & $g_{(s)}^{1,(n, 2)}$ & $g_{(s)}^{2,(n, 2)}$ & $g_{(s)}^{3,(n, 2)}$ & $g_{(s)}^{4,(n, 2)}$ \\
\hline 0 & $-\frac{n^{4}+2 E_{4}}{24}$ & $-\frac{n^{2}}{12}$ & $-\frac{1}{96}$ & 0 \\
\hline 1 & $\frac{8 n^{6}+182 n^{2} E_{4}-85 E_{6}}{5040}$ & $\frac{41 E_{4}+34 n^{4}}{2880}$ & $\frac{5 n^{2}}{576}$ & $\frac{1}{2304}$ \\
\hline 2 & $\frac{2300 E_{6} n^{2}-1722 E_{4}^{2}-1386 E_{4} n^{4}-11 n^{8}}{362880}$ & $\frac{-595 E_{4} n^{2}+290 E_{6}-52 n^{6}}{120960}$ & $\frac{-34 E_{4}-59 n^{4}}{69120}$ & $\frac{-n^{2}}{4608}$ \\
\hline 3 & $\frac{-5 E_{6}\left(3539 E_{4}+2376 n^{4}\right)+7 n^{10}}{19958400}$ & $\frac{42 E_{4}\left(10 E_{4}+9 n^{4}\right)-628 E_{6} n^{2}+7 n^{8}}{870912}$ & $\frac{217 E_{4} n^{2}-105 E_{6}+43 n^{6}}{1451520}$ & $\frac{n^{4}}{55296}$ \\
\hline
\end{tabular}

and in a similar fashion, we can tabulate the coefficients $h_{(s)}^{i,(n, 2)}$ as follows

\begin{tabular}{|c|r|r|r|r|}
\hline$s$ & $h_{(s)}^{1,(n, 2)}$ & $h_{(s)}^{2,(n, 2)}$ & $h_{(s)}^{3,(n, 2)}$ & \multicolumn{1}{c|}{$h_{(s)}^{4,(n, 2)}$} \\
\hline 0 & $\frac{\psi_{2}^{2}}{72}$ & $-\frac{\psi_{2}}{72}$ & $\frac{1}{288}$ & 0 \\
\hline 1 & $\frac{E_{6}+\psi_{2}^{3}}{432}$ & $\frac{3 E_{4}-4 \psi_{2}^{2}}{1728}$ & $\frac{\psi_{2}}{1728}$ & $-\frac{1}{6912}$ \\
\hline 2 & $\frac{8 E_{6} \psi_{2}+11 \psi_{2}^{4}}{51840}$ & $\frac{-8 E_{6}-11 \psi_{2}^{3}}{51840}$ & $\frac{5 \psi_{2}^{2}-4 E_{4}}{69120}$ & 0 \\
\hline 3 & $\frac{\psi_{2}^{3}\left(11 \psi_{2}^{2}-12 E_{4}\right)}{435456}$ & $\frac{\psi_{2}^{2}\left(12 E_{4}-11 \psi_{2}^{2}\right)}{435456}$ & $\frac{8 E_{6}+7 \psi_{2}^{3}}{1741824}$ & 0 \\
\hline
\end{tabular}




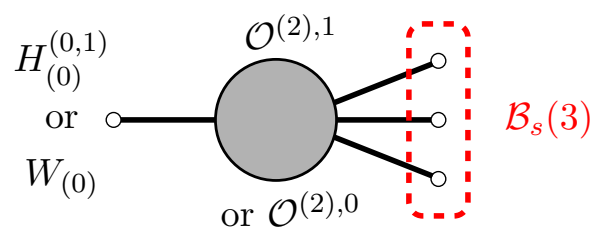

Figure 3. Coupling $H_{(0)}^{(0,1)}\left(\right.$ via $\left.\mathcal{O}^{(2), 1}\right)$ and $W_{(0)}\left(\right.$ via $\left.\mathcal{O}^{(2), 0}\right)$ to three $H_{\left(s_{i}\right)}^{(0,1)}$ comprised in $\mathcal{B}_{s}(3)$.

We can similarly treat the contribution $H_{(s)}^{(0,0,2)}$ in (3.1). Adapting the notation of [22], we have

$$
H_{(s)}^{(0,0,2)}(\rho, S)=\sum_{i=1}^{5} u_{(s)}^{i,(2,2)}(\rho) \phi_{-2,1}^{5-i}(\rho, S) \phi_{0,1}^{i-1}(\rho, S),
$$

where we find for the leading terms in $s$

\begin{tabular}{|l|r|r|r|c|c|}
\hline$s$ & $u_{(s)}^{1,(2,2)}$ & $u_{(s)}^{2,(2,2)}$ & $u_{(s)}^{3,(2,2)}$ & $u_{(s)}^{4,(2,2)}$ & $u_{(s)}^{5,(2,2)}$ \\
\hline 0 & $\frac{2 E_{2}\left(\psi_{2}^{2}-6 E_{4}\right)+10 E_{6}-\psi_{2}^{3}}{1728}$ & $\frac{\psi_{2}\left(\psi_{2}-2 E_{2}\right)-3 E_{4}}{1728}$ & $\frac{-4 E_{2}-\psi_{2}}{6912}$ & $-\frac{1}{6912}$ & 0 \\
\hline 1 & $\frac{4 E_{2}\left(7 \psi_{2}^{3}-44 E_{6}\right)+3 E_{4}\left(68 E_{4}+35 \psi_{2}^{2}\right)-42 \psi_{2}^{4}}{145152}$ & $\frac{E_{2}\left(276 E_{4}-40 \psi_{2}^{2}\right)-146 E_{6}+5 \psi_{2}^{3}}{207360}$ & $\frac{\psi_{2}\left(4 E_{2}-5 \psi_{2}\right)+18 E_{4}}{82944}$ & $\frac{4 E_{2}+\psi_{2}}{288 \cdot 24^{2}}$ & $\frac{1}{165888}$ \\
\hline
\end{tabular}

Due to their complexity, we refrain from writing higher terms explicitly.

Combining these explicit expressions suggests that $B_{(s)}^{(2,2)}$ can be presented as

$$
\begin{aligned}
B_{(s)}^{(2,2)}\left(\rho, S, \widehat{a}_{1}\right)= & \mathcal{T}_{2}\left(H_{(s)}^{(0,0,1)}\right)+2 \mathcal{O}^{(2), 1} \mathcal{H}_{2}\left[H_{(0)}^{(0,1)} H_{(s)}^{(0,1)}\right]-2^{2 s-4} K_{(s)}^{(1,2)}\left(2 \rho, 2 S, 2 \widehat{a}_{1}\right) \\
& +\left[H_{(0)}^{(0,1)} \mathcal{O}^{(2), 1}-\frac{1}{4} W_{(0)} \mathcal{O}^{(2), 0}\right] \mathcal{B}_{s}(3)+H_{(0)}^{(0,1)} \sum_{\underline{s}=\left\{s_{1}, s_{2}, s_{3}\right\}} \mathcal{O}_{2, \underline{s}}^{(2), 1} \prod_{i=1}^{3} H_{\left(s_{i}\right)}^{(0,1)},
\end{aligned}
$$

where we used the shorthand notation $\underline{s}=\left\{s_{1}, s_{2}, s_{3}\right\}$. The terms in the first line of (3.5) are obtained through particular operations from the contributions (3.2) or (3.3) (i.e. the free energy to order $Q_{R}^{1}$ ). The first term in the second line corresponds to a coupling of $H_{(0)}^{(0,0)}$ (through $\left.\mathcal{O}^{(2), 1}\right)$ or $W_{(0)}$ (through $\left.\mathcal{O}^{(2), 0}\right)$ to three $H_{\left(s_{i}\right)}^{(0,1)}$ forming $\mathcal{B}_{s}(3)$, as shown in figure 3 . The latter is built from three $H_{\left(s_{i}\right)}^{(0,1)}$ in the following fashion

$$
\mathcal{B}_{s}(3)=\frac{1}{8} \sum_{\substack{s_{1}, s_{2}, s_{3} \\
s_{1}+s_{2}+s_{3}=s+2}} c_{\underline{s}} H_{\left(s_{1}\right)}^{(0,1)} H_{\left(s_{2}\right)}^{(0,1)} H_{\left(s_{3}\right)}^{(0,1)}, \quad \text { with } \quad \begin{aligned}
& c_{\underline{s}} \in \mathbb{Z} \\
& \underline{s}=\left\{s_{1}, s_{2}, s_{3}\right\}
\end{aligned} .
$$

Explicitly, for the lowest values of $s$, we find

$$
\begin{aligned}
& \mathcal{B}_{0}(3)=0, \quad \mathcal{B}_{1}(3)=4 H_{(0)}^{(0,1)} H_{(1)}^{(0,1)} H_{(2)}^{(0,1)}-3\left(H_{(0)}^{(0,1)}\right)^{2} H_{(3)}^{(0,1)}, \\
& \mathcal{B}_{2}(3)=4\left(H_{(1)}^{(0,1)}\right)^{2} H_{(2)}^{(0,1)}+6 H_{(0)}^{(0,1)}\left(H_{(2)}^{(0,1)}\right)^{2}+12 H_{(0)}^{(0,1)} H_{(1)}^{(0,1)} H_{(3)}^{(0,1)}+2\left(H_{(0)}^{(0,1)}\right)^{2} H_{(4)}^{(0,1)}, \\
& \mathcal{B}_{3}(3)=15\left(H_{(1)}^{(0,1)}\right)^{2} H_{(3)}^{(0,1)}+H_{(0)}^{(0,1)} H_{(2)}^{(0,1)} H_{(3)}^{(0,1)}+100 H_{(0)}^{(0,1)} H_{(1)}^{(0,1)} H_{(4)}^{(0,1)}+192\left(H_{(0)}^{(0,1)}\right)^{2} H_{(5)}^{(0,1)} .
\end{aligned}
$$




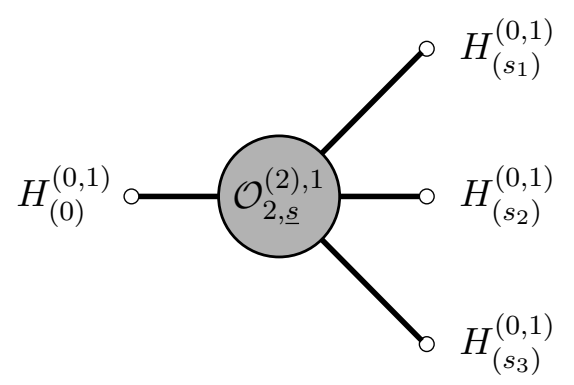

Figure 4. Coupling of $H_{(0)}^{(0,1)}$ to three $H_{\left(s_{i}\right)}^{(1,2)}$ through $\mathcal{O}_{2, \underline{s}}^{(2), 1}$.

The explicit numerical coefficients appearing in $\mathcal{B}_{s}(3)$ for higher values in $s$ are difficult to predict (and are in fact not unique), thus constituting information encoded in the free energy, that does not immediately follow from the case $N=1$. Finally, the last line in (3.5) represents new terms that involve similar sums as in (3.2), with higher powers in $n$, as well as Eisenstein series. It is, however, amusing that this contribution can still be represented in a diagrammatic fashion along the lines of the remaining terms: as shown in figure 4, it can be represented as $H_{(0)}^{(0,0)}$ coupling to three other $H_{\left(s_{i}\right)}^{(0,1)}$ through the coupling function $\mathcal{O}_{2, \underline{s}}^{(2), 1} \cdot{ }^{5}$ For given $s$, the latter is

$$
\mathcal{O}_{2, \underline{s}}^{(2), 1}=\sum_{n=1}^{\infty} \frac{d_{s ; \underline{s}} n^{\kappa}}{1-Q_{\rho}^{n}}\left(Q_{\widehat{a}_{1}}^{n}+\frac{Q_{\rho}^{n}}{Q_{\widehat{a}_{1}}^{n}}\right)+\mathfrak{w}_{s, \underline{s}}^{r=2}(\rho) .
$$

In the first term, we have $s=\frac{\kappa-5}{2}+s_{1}+s_{2}+s_{3}$ and the $d_{s ; \underline{s}} \in \mathbb{Q}$ are numerical coefficients. The second term in (3.7) is independent of $\widehat{a}_{1}$ and $\mathfrak{w}_{s, \underline{s}}^{r=2}(\rho)$ are modular forms of weight $w$, which satisfy $s_{1}+s_{2}+s_{3}+\frac{w}{2}=s+3$. To leading order in $s$, we find explicitly

\begin{tabular}{|c|c|r|r|}
\hline$s$ & $\underline{s}$ & $d_{s ; \underline{s}}$ & $\mathfrak{w}_{s, \underline{s}}^{2}$ \\
\hline 0 & $\{0,0,1\}$ & 2 & 0 \\
\hline & $\{0,0,0\}$ & $\frac{-1}{24}$ & 0 \\
\hline 1 & $\{0,1,1\}$ & 5 & $\frac{-4 E_{4}}{15}$ \\
\hline & $\{0,0,2\}$ & $\frac{26}{3}$ & $\frac{-10 E_{4}}{63}$ \\
\hline & $\{0,0,1\}$ & $\frac{-17}{60}$ & $\frac{19 E_{6}}{630}$ \\
\hline & $\{0,0,0\}$ & $\frac{1}{630}$ & 0 \\
\hline 2 & $\{1,1,1\}$ & 3 & $\frac{-4 E_{4}}{15}$ \\
\hline & $\{0,1,2\}$ & $\frac{85}{3}$ & 0 \\
\hline
\end{tabular}

\begin{tabular}{|r|c|r|r|}
\hline$s$ & $\underline{s}$ & $d_{s ; \underline{s}}$ & $\mathfrak{w}_{s, \underline{s}}^{2}$ \\
\hline 2 & $\{0,0,3\}$ & $\frac{115}{3}$ & $\frac{14 E_{4}}{15}$ \\
\hline & $\{0,1,1\}$ & $\frac{-59}{120}$ & $\frac{-E_{6}}{105}$ \\
\hline & $\{0,0,2\}$ & $\frac{-11}{12}$ & 0 \\
\hline & $\{0,0,1\}$ & $\frac{13}{1260}$ & $\frac{E_{4}^{2}}{1260}$ \\
\hline & $\{0,0,0\}$ & $\frac{-11}{362880}$ & 0 \\
\hline 3 & $\{1,1,2\}$ & $\frac{62}{3}$ & $\frac{-10 E_{4}}{7}$ \\
\hline & $\{0,1,3\}$ & $\frac{314}{3}$ & 0 \\
\hline & $\{0,0,4\}$ & 254 & 0 \\
\hline
\end{tabular}

\begin{tabular}{|c|c|r|r|}
\hline$s$ & $\underline{s}$ & $d_{s ; \underline{s}}$ & $\mathfrak{w}_{s, \underline{s}}^{2}$ \\
\hline 3 & $\{1,1,1\}$ & $\frac{-1}{4}$ & $\frac{-5 E_{6}}{126}$ \\
\hline & $\{0,1,2\}$ & $\frac{-5}{2}$ & $\frac{61 E_{6}}{1386}$ \\
\hline & $\{0,0,3\}$ & $\frac{-18}{5}$ & $\frac{91 E_{6}}{990}$ \\
\hline & $\{0,1,1\}$ & $\frac{43}{2520}$ & 0 \\
\hline & $\{0,0,2\}$ & $\frac{41}{1260}$ & $\frac{5 E_{4}^{2}}{616}$ \\
\hline & $\{0,0,1\}$ & $\frac{-1}{5184}$ & 0 \\
\hline & $\{0,0,0\}$ & $\frac{1}{2851200}$ & 0 \\
\hline
\end{tabular}

\footnotetext{
${ }^{5}$ Our notation is explained in section 5: we denote $\mathcal{O}_{r, s}^{(N), i}$, where $i$ is a summation index labelling different classes of couplings, $r$ denotes the order of $Q_{R}^{r}$, while $\underline{s}=\left\{s_{1}, \ldots, s_{k}\right\}$ is a set of labels governing the $\epsilon$-expansion.
} 


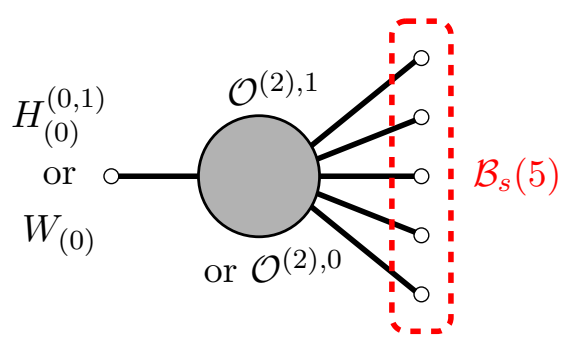

Figure 5. Coupling $H_{(0,0)}^{(0,1)}\left(\operatorname{via} \mathcal{O}^{(2), 1}\right)$ and $W_{(0)}\left(\right.$ via $\left.\mathcal{O}^{(2), 0}\right)$ to five $H_{\left(s_{i}\right)}^{(0,1)}$ comprised in $\mathcal{B}_{s}(5)$.

We stress that the $d_{s ; \underline{s}}$ and $\mathfrak{w}_{s, \underline{s}}^{2}$ are in general not unique ${ }^{6}$ and the above table simply gives a(n economic) presentation. We finally remark that, despite being structurally very similar, we have chosen to present the terms involving $\mathcal{O}^{(2), 0,1}$ and $\mathcal{O}_{2, \underline{s}}^{(2), 1}$ separately in the last line of (3.5) since the latter is a completely holomorphic function, while the former (once the $\mathcal{O}^{(2), 1}$ are summed up) also contain quasi-modular contributions (see eq. (5.4) below). From a 'Feynman diagrammatic' point of view, however, these two represent two similar classes of couplings.

\section{$3.3 \quad$ Order $Q_{R}^{3}$}

We can continue the previous analysis to order $Q_{R}^{3}$. However, since explicit expansions to this order are very difficult to compute, we shall limit ourselves to only the leading orders in $s$. Due to their complexity, we refrain from explicitly writing the $H_{(s)}^{(n, 0,3)}(\rho, S)$, but we refer the reader to [22]. The leading two orders in $s$, however, suggest the following presentation

$$
\begin{aligned}
B_{(s)}^{(2,3)}\left(\rho, S, \widehat{a}_{1}\right) & =\mathcal{T}_{3}\left(H_{(s)}^{(0,0,1)}\right)+3 \mathcal{O}^{(2), 1} \mathcal{H}_{3}\left[H_{(0)}^{(0,1)} H_{(s)}^{(0,1)}\right]-3^{2 s-4} K_{(s)}^{(1,2)}\left(3 \rho, 3 S, 3 \widehat{a}_{1}\right) \\
& +\left[H_{(0)}^{(0,1)} \mathcal{O}^{(2), 1}-\frac{1}{9} W_{(0)} \mathcal{O}^{(2), 0}\right] \mathcal{B}_{s}(5)+H_{(0)}^{(0,1)} \sum_{\underline{s}=\left\{s_{1}, s_{2}, s_{3}, s_{4}, s_{5}\right\}} \mathcal{O}_{3, \underline{s}}^{(2), 1} \prod_{i=1}^{5} H_{\left(s_{i}\right)}^{(0,1)},
\end{aligned}
$$

which directly generalises (3.5) to order $Q_{R}^{3}$ : the terms in the first line are obtained through certain operators from the free energy at order $Q_{R}^{1}$. Furthermore, the first term in the second line of $(3.8)$ couples $H_{(0)}^{(0,1)}$ (through $\left.\mathcal{O}^{(2), 1}\right)$ and $W_{(0)}\left(\right.$ through $\left.\mathcal{O}^{(2), 0}\right)$ to $\mathcal{B}_{s}(5)$, as schematically shown in figure 5 . To leading order in $s$, we find for the former:

$$
\begin{aligned}
\mathcal{B}_{0}(5)= & 0 \\
\mathcal{B}_{1}(5)= & \frac{64}{11} H_{(0)}^{(0,1)}\left[22\left(H_{(1)}^{(0,1)}\right)^{3} H_{(2)}^{(0,1)}\right. \\
& +99 H_{(0)}^{(0,1)}\left(H_{(1)}^{(0,1)}\right)^{2} H_{(3)}^{(0,1)}+103\left(H_{(0)}^{(0,1)}\right)^{2} H_{(2)}^{(0,1)} H_{(3)}^{(0,1)} \\
& \left.+330\left(H_{(0)}^{(0,1)}\right)^{2} H_{(1)}^{(0,1)} H_{(4)}^{(0,1)}\right] .
\end{aligned}
$$

\footnotetext{
${ }^{6}$ This is e.g. due to linear relations such as e.g. $E_{4} H_{(0)}^{(0,1)}=240 H_{(2)}^{(0,1)}$.
} 


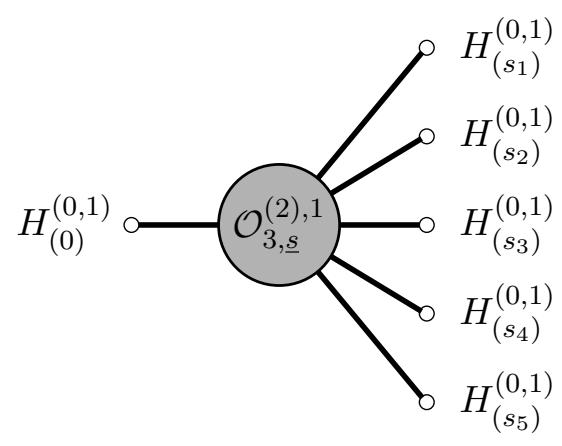

Figure 6. Coupling of $H_{(0)}^{(0,1)}$ to five $H_{\left(s_{i}\right)}^{(1,2)}$ through $\mathcal{O}_{3, \underline{s}}^{(2), 1}$.

Finally, the last term in the second line in (3.8) generalises the contribution depicted in figure 4 in the sense that it couples $H_{(0)}^{(0,1)}$ to five different $H_{\left(s_{i}\right)}^{(0,1)}$ (rather than just 3 ) through a coupling function $\mathcal{O}_{2, \underline{s}}^{(2), 1}$, where $\underline{s}=\left\{s_{1}, s_{2}, s_{3}, s_{4}, s_{5}\right\}$, as schematically shown in figure 6 . We can write

$$
\mathcal{O}_{3, \underline{s}}^{(2), 1}=\sum_{n=1}^{\infty} \frac{d_{s ; \underline{\underline{s}}} n^{\kappa}}{1-Q_{\rho}^{n}}\left(Q_{\widehat{a}_{1}}^{n}+\frac{Q_{\rho}^{n}}{Q_{\widehat{a}_{1}}^{n}}\right)+\mathfrak{w}_{s, \underline{s}}^{r=2}(\rho),
$$

where $d_{s, \underline{s}} \in \mathbb{Q}$ are numerical coefficient and we have $s=\frac{\kappa-9}{2}+\sum_{a=1}^{5} s_{a}$. Furthermore, the $\mathfrak{w}_{s, \underline{s}}^{r=2}(\rho)$ are independent of $\widehat{a}_{1}$ and are modular forms of weight $w$, which satisfy $\sum_{a=1}^{5} s_{a}+\frac{w}{2}=s+5$. The leading contributions in $s$ can be tabulated in the following:

\begin{tabular}{|r|c|r|r|}
\hline$s$ & $\underline{s}$ & $d_{s, \underline{s}}$ & $\mathfrak{w}_{s, \underline{s}}^{r=2}$ \\
\hline 0 & $\{0,0,1,1,1\}$ & $-\frac{32}{2}$ & 0 \\
\hline & $\{0,0,0,1,2\}$ & -64 & 0 \\
\hline & $\{0,0,0,0,3\}$ & $-\frac{176}{3}$ & 0 \\
\hline & $\{0,0,0,1,1\}$ & $\frac{4}{3}$ & 0 \\
\hline & $\{0,0,0,0,2\}$ & $\frac{14}{9}$ & 0 \\
\hline & $\{0,0,0,0,1\}$ & $-\frac{4}{135}$ & 0 \\
\hline & $\{0,0,0,0,0\}$ & $\frac{1}{7560}$ & 0 \\
\hline 1 & $\{0,1,1,1,1\}$ & $-\frac{80}{3}$ & $-\frac{16}{5} E_{4}$ \\
\hline & $\{0,0,1,1,2\}$ & $-\frac{1264}{3}$ & $-\frac{32}{21} E_{4}$ \\
\hline
\end{tabular}

\begin{tabular}{|r|c|r|r|}
\hline$s$ & $\underline{s}$ & $d_{s, \underline{s}}$ & $\mathfrak{w}_{s, \underline{s}}^{r=2}$ \\
\hline & $\{0,0,0,2,2\}$ & $-\frac{5504}{9}$ & $\frac{2000}{693} E_{4}$ \\
\hline & $\{0,0,0,1,3\}$ & $-\frac{2960}{3}$ & $\frac{1024}{165} E_{4}$ \\
\hline & $\{0,0,1,1,1\}$ & $\frac{104}{15}$ & $-\frac{32}{315} E_{6}$ \\
\hline & $\{0,0,0,1,2\}$ & 30 & 0 \\
\hline & $\{0,0,0,0,3\}$ & $\frac{908}{45}$ & $\frac{56}{495} E_{6}$ \\
\hline & $\{0,0,0,1,1\}$ & $-\frac{34}{105}$ & 0 \\
\hline & $\{0,0,0,0,2\}$ & $-\frac{284}{945}$ & 0 \\
\hline & $\{0,0,0,0,1\}$ & $\frac{1}{280}$ & 0 \\
\hline & $\{0,0,0,0,0\}$ & $-\frac{4}{467775}$ & 0 \\
\hline
\end{tabular}

As in the case of $\mathcal{O}_{2, \underline{s}}^{(2), 1}$, however, we stress that the above coefficients are not unique, but just represent an economic choice.

\subsection{Nekrasov-Shatashvili limit}

The discussion above was exclusively focused on the so-called unrefined limit, i.e. $\epsilon_{1}=-\epsilon_{2}$. For completeness, we also briefly comment on another limit, namely the NekrasovShatashvili limit [23, 24], i.e. effectively $\epsilon_{2} \rightarrow 0$. However, we shall limit ourselves to 
studying the order $Q_{R}^{1}$ (up to order $\epsilon_{1}^{5}$ ): while the latter also exhibits some very interesting patterns, extracting a coupling function akin to (3.2) (in the unrefined limit) is more involved. We shall therefore leave an in-depth analysis of this limit to future work [54].

We start with a presentation of the free energy (2.3) like in (3.1), suitable for the NS-limit

$B_{(s, 0)}^{(2, r)}=H_{(s, 0)}^{(0,0, r)}(\rho, S)+K_{(s, 0)}^{(r, 2)}\left(\rho, S, \widehat{a}_{1}\right), \quad$ with $\quad K_{(s, 0)}^{(1,2)}=\sum_{n=1}^{\infty} H_{(s, 0)}^{(n, 0, r)}(\rho, S)\left(Q_{\widehat{a}_{1}}^{n}+\frac{Q_{\rho}^{n}}{Q_{\widehat{a}_{1}}^{n}}\right)$.

The contributions $H_{(s, 0)}^{(0,0, r)}$ (see $(2.5)$ ) have been studied in [25] and have been shown to exhibit a very particular Hecke symmetry. We therefore focus on the contribution $K_{(s, 0)}^{(r, 2)}\left(\rho, S, \widehat{a}_{1}\right)$, or more precisely $H_{(s, 0)}^{(n, 0, r)}$. In order to reveal similar structures as in the unrefined limit (see eq. (3.2)) we first need to compute the $H_{(s, 0)}^{(0,1)}$. The latter can be written as $[22]$

$$
H_{(s, 0)}^{(0,1)}=u_{(s, 0)}^{1,(1,1)}(\rho) \phi_{-2,1}(\rho, S)+u_{(s, 0)}^{2,(1,1)}(\rho) \phi_{0,1}(\rho, S),
$$

where $u_{(s, 0)}^{i,(1,1)}(\rho)$ are quasi-modular forms of weight $s$ (for $\left.i=1\right)$ and $s-2$ (for $i=2$ ). For low values of $s$, they can be tabulated as follows

\begin{tabular}{|c|r|r|r|r|}
\hline$s$ & 0 & 2 & 4 & 6 \\
\hline$u_{(s, 0)}^{1,(1,1)}$ & -1 & $-\frac{E_{2}}{48}$ & $-\frac{5 E_{2}^{2}+13 E_{4}}{40 \cdot 24^{2}}$ & $-\frac{184 E_{6}+273 E_{2} E_{4}+35 E_{2}^{3}}{70 \cdot 24^{4}}$ \\
\hline$u_{(s, 0)}^{2,(1,1)}$ & 0 & $\frac{1}{96}$ & $\frac{E_{2}}{8 \cdot 24^{2}}$ & $\frac{7 E_{4}+5 E_{2}^{2}}{160 \cdot 24^{3}}$ \\
\hline
\end{tabular}

Notice that the $H_{(s, 0)}^{(0,1)}$ satisfy

$$
\frac{\partial H_{(s, 0)}^{(0,1)}}{\partial E_{2}}=\frac{1}{2 \cdot 24} H_{(s-1,0)}^{(0,1)}
$$

Furthermore, the $H_{(s, 0)}^{(n, 0, r=1)}$ (up to $s=6$ ) have been computed in [22] and are of the form

$$
H_{(s, 0)}^{(n, 0,2)}=\sum_{i=1}^{2} g_{(s, 0)}^{i,(n, 1)} \frac{\phi_{-2,1}^{3-i} \phi_{0,1}^{i-1}}{1-Q_{\rho}^{n}}, \quad \text { with } \quad g_{(s, 0)}^{i,(n, 1)}=\sum_{\kappa \in \mathbb{N}_{\text {odd }}} p_{\kappa, s}^{i}(\rho) n^{\kappa}
$$

where $p_{\kappa, s}^{i}(\rho)$ are quasi-modular forms of weight $s-\kappa-2 i+3$, which can be tabulated as follows

\begin{tabular}{|c|c|c|c|c|c|c|c|c|c|c|}
\hline$s$ & 0 & \multicolumn{2}{|c|}{2} & \multicolumn{3}{|c|}{4} & \multicolumn{3}{|c|}{6} \\
\hline$\kappa$ & 1 & 3 & 1 & 5 & 3 & 1 & 7 & 5 & 3 & 1 \\
\hline$p_{\kappa, s}^{1}$ & -2 & $\frac{1}{3}$ & $\frac{-E_{2}}{12}$ & $\frac{-1}{60}$ & $\frac{E_{2}}{72}$ & $\frac{-13 E_{4}-10 E_{2}^{2}}{5760}$ & $\frac{192}{35 \cdot 24^{3}}$ & $\frac{-48 E_{2}}{5 \cdot 24^{3}}$ & $\frac{2\left(10 E_{2}^{2}+13 E_{4}\right)}{5 \cdot 24^{3}}$ & $\frac{-92 E_{6}-273 E_{4} E_{2}-70 E_{2}^{3}}{210 \cdot 24^{3}}$ \\
\hline$p_{\kappa, s}^{2}$ & 0 & 0 & $\frac{1}{24}$ & 0 & $\frac{-1}{144}$ & $\frac{E_{2}}{576}$ & 0 & $\frac{1}{5 \cdot 24^{2}}$ & $\frac{-E_{2}}{6 \cdot 24^{2}}$ & $\frac{5\left(E_{4}+E_{2}^{2}\right)}{2 \cdot 24^{3}}$ \\
\hline$p_{\kappa, s}^{3}$ & 0 & 0 & 0 & 0 & 0 & $\frac{-1}{8 \cdot 24^{2}}$ & 0 & 0 & $\frac{1}{2 \cdot 24^{3}}$ & $\frac{-E_{2}}{8 \cdot 24^{3}}$ \\
\hline
\end{tabular}


Using this explicit form, we find that $K_{(s, 0)}^{(1,2)}$ can also be written as

$K_{(s, 0)}^{(1,2)}=\sum_{a, b=0}^{s / 2} H_{(a, 0)}^{(0,1)} \mathcal{M}_{a b}\left(\widehat{a}_{1}\right) H_{(b, 0)}^{(0,1)}, \quad$ with $\quad \mathcal{M}_{a b}=-2 \sum_{n=1}^{\infty} \frac{(-1)^{\frac{s}{2}+a+b} n^{s+1-2(a+b)}\left(Q_{\widehat{a}_{1}}^{n}+\frac{Q_{\rho}^{n}}{Q_{\widehat{a}_{1}}^{n}}\right)}{\left(1-Q_{\rho}^{n}\right) \Gamma(s-2(a+b-1))}$

Here, using a slightly imprecise notation, we understand that $1 / \Gamma(-m)=0 \quad \forall m \in \mathbb{N} \cup\{0\}$. In this way, $\mathcal{M}$ is a $\left(\frac{s}{2}+1\right) \times\left(\frac{s}{2}+1\right)$ symmetric matrix with zero entries for $a+b>\frac{s}{2}$. Furthermore, the entries on the off-diagonal of $\mathcal{M}$ correspond precisely to the coupling function $\mathcal{O}^{(2), 1}$ in (3.2), while all entries in the top-left half correspond to holomorphic derivatives of the latter with respect to $\widehat{a}_{1}$. While this is still a very intriguing pattern (which shall be further analysed in [54]) it does not make the coupling function appear in such a clean fashion as in the unrefined case (see eq. (3.2)). Therefore, in the following we shall further analyse the latter limit.

\section{Example $N=3$}

Following [21, 22], the free energy for $N=3$ can be decomposed as in (3.1) for the case $N=2$

$$
B_{(s)}^{(3, r)}\left(\rho, S, \widehat{a}_{1}, \widehat{a}_{2}\right)=H_{(s)}^{(0,0,0, r)}(\rho, S)+K_{(s)}^{(r, 3)}\left(\rho, S, \widehat{a}_{1}, \widehat{a}_{2}\right)
$$

where in the following we shall limit ourselves to $r=1$. The contribution $K_{(s)}^{(r, 3)}$ comprises three different types of terms. Using a slightly different notation than in [22] and generalising a pattern arising up to order $s=2$, we can present it in the following form

$$
\begin{aligned}
& K_{(s)}^{(1,3)}\left(\rho, S, \widehat{a}_{1}, \widehat{a}_{2}\right)=\sum_{n=1}^{\infty}\left[n \frac{\lambda_{s}^{1}(\rho, S)}{1-Q_{\rho}^{n}}\left(\Delta_{+}^{(n)}+Q_{\rho}^{n} \Delta_{+}^{(-n)}\right)+n^{2} \frac{\lambda_{s}^{2}(\rho, S)}{\left(1-Q_{\rho}^{n}\right)^{2}}\left(\Delta_{+}^{(n)}+\Delta_{+}^{(-n)}\right)\right] \\
& +\frac{\lambda_{s}^{3}(\rho, S)}{24} \sum_{n_{1}, n_{2}=1}^{\infty}\left[\frac{n_{2}\left(n_{2}+2 n_{1}\right)}{\left(1-Q_{\rho}^{n_{1}}\right)\left(1-Q_{\rho}^{n_{2}}\right)}+\frac{n_{1}^{2}-n_{2}^{2}}{\left(1-Q_{\rho}^{n_{1}}\right)\left(1-Q_{\rho}^{n_{1}+n_{2}}\right)}\right] \sum_{i=1}^{3} Q_{\widehat{a}_{i}}^{n_{1}+n_{2}} \sum_{j \neq i} Q_{\widehat{a}_{j}}^{n_{1}} .
\end{aligned}
$$

In the second line we have defined $Q_{\widehat{a}_{3}}=Q_{\rho} /\left(Q_{\widehat{a}_{1}} Q_{\widehat{a}_{2}}\right)$ to keep the notation compact, while $\Delta_{+}^{(n)}=\sum_{i=1}^{3} Q_{\widehat{a}_{i}}^{n}$. Furthermore, the $\lambda_{s}^{1,2,3}$ are quasi-Jacobi forms of index 3 and weights ${ }^{7}$ $w_{1}=2 s-4, w_{2}=w_{3}=2 s-6$, respectively. The $\lambda_{s}^{i}$ can be decomposed as

$$
\lambda_{s}^{i}(\rho, S)=-\sum_{a=1}^{3} \alpha_{a, s}^{i}(\rho)\left(\phi_{-2,1}(\rho, S)\right)^{4-a}\left(\phi_{0,1}(\rho, S)\right)^{a-1},
$$

\footnotetext{
${ }^{7}$ We remark that the $\lambda_{s}^{i}$ are independent of the summation variables $n$ or $n_{1,2}$ in (4.2).
} 


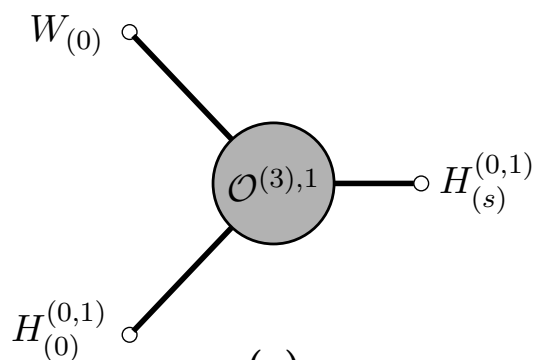

(a)

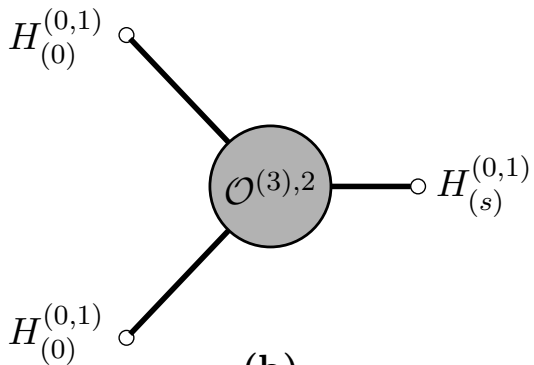

(b)

Figure 7. Schematic contributions to $K_{(s)}^{(1,3)}$, in the form of a single $H_{(0)}^{(0,1)}$ and a single $W_{(0)}$ (diagram (a)) or two $H_{(0)}^{(0,1)}$ (diagram (b)) coupling to $H_{(s)}^{(0,1)}$.

where the $\alpha_{a, s}^{i}$ are quasi-modular forms of weight $w_{i}+6$ (for $a=1$ ), $w_{i}+4$ (for $a=2$ ) and $w_{i}+2$ (for $a=3$ ) respectively. Up to order $s=3$, they can be tabulated as follows

\begin{tabular}{|c|c|c|c|c|c|c|c|c|c|c|c|c|c|c|c|}
\hline$s$ & \multicolumn{3}{|c|}{0} & \multicolumn{3}{|c|}{1} & \multicolumn{3}{c|}{2} & \multicolumn{3}{|c|}{3} & \multicolumn{3}{|c|}{4} \\
\hline$a$ & 1 & 2 & 3 & 1 & 2 & 3 & 1 & 2 & 3 & 1 & 2 & 3 & 1 & 2 & 3 \\
\hline$\alpha_{a, s}^{1}$ & $\frac{E_{2}}{6}$ & $\frac{1}{12}$ & 0 & 0 & $-\frac{E_{2}}{144}$ & $-\frac{1}{288}$ & $\frac{E_{2} E_{4}}{1440}$ & $\frac{E_{4}}{2880}$ & 0 & $\frac{E_{2} E_{6}}{36288}$ & $\frac{E_{6}}{72576}$ & 0 & $\frac{E_{2} E_{4}^{2}}{1036800}$ & $\frac{E_{4}^{2}}{2073600}$ & 0 \\
\hline$\alpha_{a, s}^{2}$ & 1 & 0 & 0 & 0 & $-\frac{1}{24}$ & 0 & $\frac{E_{4}}{240}$ & 0 & 0 & $\frac{E_{6}}{6048}$ & 0 & 0 & $\frac{E_{4}^{2}}{172800}$ & 0 & 0 \\
\hline$\alpha_{a, s}^{3}$ & 24 & 0 & 0 & 0 & -1 & 0 & $\frac{E_{4}}{10}$ & 0 & 0 & $\frac{E_{6}}{252}$ & 0 & 0 & $\frac{E_{4}^{2}}{7200}$ & 0 & 0 \\
\hline
\end{tabular}

These contributions can be summarised by the following pattern

$$
K_{(s)}^{(1,3)}=\mathcal{O}^{(3), 1} W_{(0)} H_{(0)}^{(0,1)} H_{(s)}^{(0,1)}+\mathcal{O}^{(3), 2} H_{(0)}^{(0,1)} H_{(0)}^{(0,1)} H_{(s)}^{(0,1)},
$$

where we defined

$$
\begin{aligned}
& \mathcal{O}^{(3), 1}=-\sum_{n=1}^{\infty} \frac{2 n}{1-Q_{\rho}^{n}}\left[\Delta_{+}^{(n)}+Q_{\rho}^{n} \Delta_{+}^{(-n)}\right], \\
& \mathcal{O}^{(3), 2}=\sum_{n_{1,2}=1}^{\infty} \frac{\sum_{i} Q_{\widehat{a}_{i}}^{n_{1}+n_{2}} \sum_{j \neq i} Q_{\widehat{a}_{j}}^{n_{1}}}{1-Q_{\rho}^{n_{1}}}\left[\frac{n_{2}\left(n_{2}+2 n_{1}\right)}{\left(1-Q_{\rho}^{n_{2}}\right)}+\frac{n_{1}^{2}-n_{2}^{2}}{\left(1-Q_{\rho}^{n_{1}+n_{2}}\right)}\right]+\sum_{n=1}^{\infty} \frac{n^{2} Q_{\rho}^{n}\left(\Delta_{+}^{(n)}+\Delta_{+}^{(-n)}\right)}{\left(1-Q_{\rho}^{n}\right)^{2}},
\end{aligned}
$$

which can be presented as either a $H_{(0)}^{(0,1)}$ and a $W_{(0)}$ or two $H_{(0)}^{(0,1)}$ coupling to $H_{(s)}^{(0,1)}$ (figure 7).

It remains to study $H_{(s)}^{(0,0,0,1)}$ appearing in $(4.1)$, for which the first few examples (in s) can be written as follows

$$
\begin{aligned}
H_{(0)}^{(0,0,0,1)} & =-\frac{1}{192} \phi_{-2,1}\left(\phi_{0,1}+2 E_{2} \phi_{-2,1}\right)^{2}, \\
H_{(1)}^{(0,0,0,1)} & =\frac{1}{4608} \phi_{0,1}\left(\phi_{0,1}+2 E_{2} \phi_{-2,1}\right)^{2}, \\
H_{(2)}^{(0,0,0,1)} & =-\frac{1}{46080} E_{4} \phi_{-2,1}\left(\phi_{0,1}+2 E_{2} \phi_{-2,1}\right)^{2}, \\
H_{(3)}^{(0,0,0,1)} & =-\frac{1}{1161216} E_{6} \phi_{-2,1}\left(\phi_{0,1}+2 E_{2} \phi_{-2,1}\right)^{2}, \\
H_{(4)}^{(0,0,0,1)} & =-\frac{1}{33177600} E_{4}^{2} \phi_{-2,1}\left(\phi_{0,1}+2 E_{2} \phi_{-2,1}\right)^{2},
\end{aligned}
$$




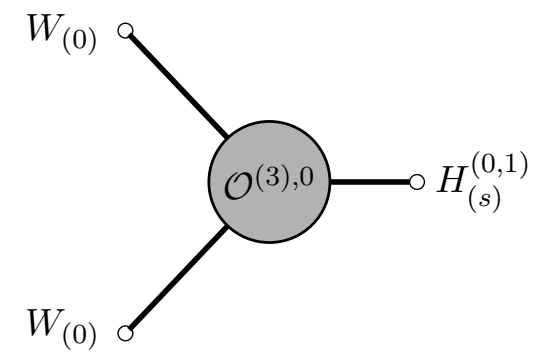

Figure 8. Contribution to $H_{(s)}^{(0,0,0,1)}$ as two $W_{(0)}$ coupling to $H_{(s)}^{(0,1)}$.

These explicit examples exhibit a clear pattern which suggest that for generic $s$ we can write

$$
H_{(s)}^{(0,0,0,1)}=\mathcal{O}^{(3), 0} W_{(0)} W_{(0)} H_{(s)}^{(0,1)}, \quad \text { where } \quad \mathcal{O}^{(3), 0}=3 .
$$

This form can graphically be represented as in figure 8, with two $W_{(0)}$ coupling through the constant $\mathcal{O}^{(3), 0}=3$ to $H_{(s)}^{(0,1)}$.

\section{General structure of the unrefined free energy}

\subsection{Summary of examples and general conjecture}

Studying explicit expansions of the free energy for the cases $N=2$ and $N=3$ in the unrefined limit has revealed a number of very intriguing patterns, which we conjecture to hold in general: we have seen that the coefficient functions $H_{(s)}^{(0,1)}$, which appear in the expansion of the free energy for $N=1$ at order $Q_{R}^{1}$ (see eq. (2.9)) serve as the fundamental building blocks to construct the free energies for $N>1$ (also to higher orders in $Q_{R}$ ). In particular we have seen that the way in which these building blocks are combined, has a certain resemblance of a (Feynman) diagrammatic expansion. More specifically, the examples studied above suggest that to leading order in $Q_{R}$, the free energy (2.6) in the unrefined limit can be written as

$$
B_{(s)}^{(N, r)}=H_{(s)}^{(0,1)}(\rho, S) \sum_{i=0}^{N-1}\left(W_{(0)}(\rho, S)\right)^{N-1-i}\left(H_{(0)}^{(0,1)}(\rho, S)\right)^{i} \mathcal{O}^{(N), i}\left(\widehat{a}_{1, \ldots, N-1}, \rho\right),
$$

where each term in this sum can be represented graphically through a diagram of the type shown in figure 9: they correspond to the 'coupling' of $i$ factors of $H_{(0)}^{(0,1)}$ and $(N-1-i)$ factors of $W_{(0)}$ to $H_{(s)}^{(0,1)}$ through $\mathcal{O}^{(N), i}\left(\widehat{a}_{1, \ldots, N-1}, \rho, S\right)$. In this decomposition, the only $s$-dependence appears through $H_{(s)}^{(0,1)}$ (on the right hand side of figure 9 ), while the only dependence on the roots $\widehat{a}_{1, \ldots, N-1}$ of the gauge algebra $\mathfrak{a}_{N+1}$ is located in $\mathcal{O}^{(N), i}$ for $i>0$. Indeed, the summand $i=0$ captures the contribution called $H_{(s)}^{(0, \ldots, 0,1)}$ in (3.1) for $N=2$ and (4.1) for $N=3$, which is independent of the $\widehat{a}_{1, \ldots, N-1}$. In general, extrapolating the examples $^{8}$ encountered in sections 3 and 4 , the $\mathcal{O}^{(N), i}$ can schematically be written in the

\footnotetext{
${ }^{8}$ In [22] further results have been published for the case $N=4$, which are also compatible with this form.
} 


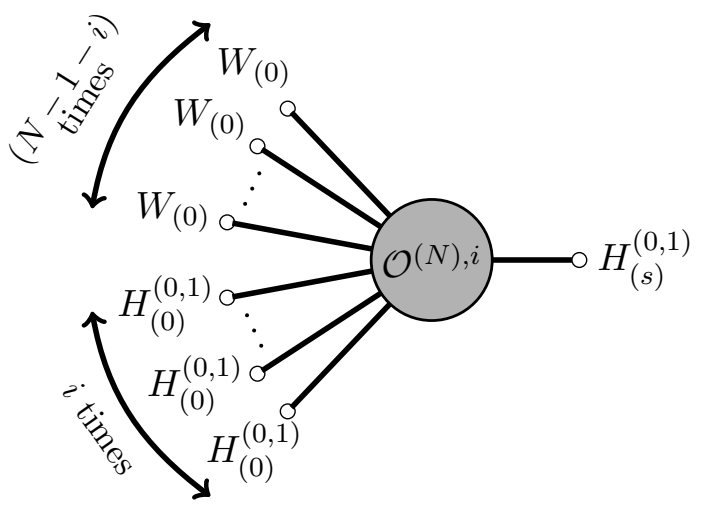

Figure 9. Schematic contribution to $B_{(s)}^{(N, 1)}$ as $(N-1-i) W_{(0)}$ and $i H_{(0)}^{(0,1)}$ coupling to $H_{(s)}^{(0,1)}$ through $\mathcal{O}^{(N), i}$, for $i=0, \ldots, N-1$.

following form

$$
\mathcal{O}^{(N), i}\left(\widehat{a}_{1, \ldots, N-1}, \rho, S\right)=\sum_{\ell} \sum_{n_{1}, \ldots, n_{i}=1}^{\infty} \frac{p_{\ell}^{i}\left(n_{1}, \ldots, n_{i}\right) \Lambda_{\ell}^{i}\left(\widehat{a}_{1}, \ldots, \widehat{a}_{N-1}, \rho ; n_{1}, \ldots, n_{i}\right)}{\prod_{a=1}^{i}\left(1-Q_{\rho}^{t_{\ell}^{i}\left(n_{1}, \ldots, n_{i}\right)}\right)} .
$$

Here $p_{\ell}^{i}$ are a set of $\ell$ homogeneous polynomials of order $i$ in $n_{1, \ldots, i}$ while $t_{\ell}^{i}$ are a set of $\ell$ linear functions in $n_{1, \ldots, i}$. Furthermore, the $\Lambda_{\ell}^{i}$ are rational functions in $Q_{\widehat{a}_{1, \ldots, i}}=e^{2 \pi i \widehat{a}_{i}}$ and $Q_{\rho}$. They can in general be written as combinations of sums over (parts of) the root lattice of the algebra $\widehat{\mathfrak{a}}_{N+1}$. Finally, in the notation in (5.2) it is understood that $\mathcal{O}^{(N), i=0}=$ const., and in fact the examples we have studied explicitly suggest ${ }^{9}$

$$
\mathcal{O}^{(N), i=0}=N,
$$

which is in agreement with the proposed T-duality of little string theories [17]. While in this work, we have only studied higher orders in $Q_{R}$ for $N=2$, it is already clear from this example that the former exhibits more complicated structures, generalising figure 9 in several ways. On the one hand side, to order $Q_{R}^{r}$, we find terms that can be obtained from the free energy at order $Q_{R}^{1}$ in (5.1) through the action of Hecke operators or through multiplying the arguments by suitable integers. Examples of this type in $N=2$ are given written in (3.5) and (3.8). Furthermore, we also expect to find new terms, which can schematically be represented as in figure 10. The latter correspond to coupling $(N-1-i)$ factors of $W_{(0)}$ and $i$ factors of $H_{(0)}^{(0,1)}$ (for $\left.i=0, \ldots, N-1\right)$ to $k=N(r-1)+1$ factors $H_{\left(s_{a}\right)}^{(0,1)}$ where $a=1, \ldots, k$. Here the coupling functions $\mathcal{O}_{r, s}^{(N), i}$ a priori are different for distinct choices of the external states $\left(H_{\left(s_{1}\right)}^{(0,1)}, \ldots, H_{\left(s_{k}\right)}^{(0,1)}\right)$. They are therefore labelled by the set of integers $\underline{s}=\left\{s_{1}, \ldots, s_{k}\right\}$. Contributions of the type shown in figure 10 for $N=2$ are exhibited in (3.7) for $r=2$ and (3.10) for $r=3$. Since they are difficult to analyse (and we have in fact not been able to determine a general pattern), we shall content ourselves with the schematic representation in figure 10 and shall not discuss them further in this work.

\footnotetext{
${ }^{9}$ We have also verified this for $N=4$ up to order $s=4$.
} 


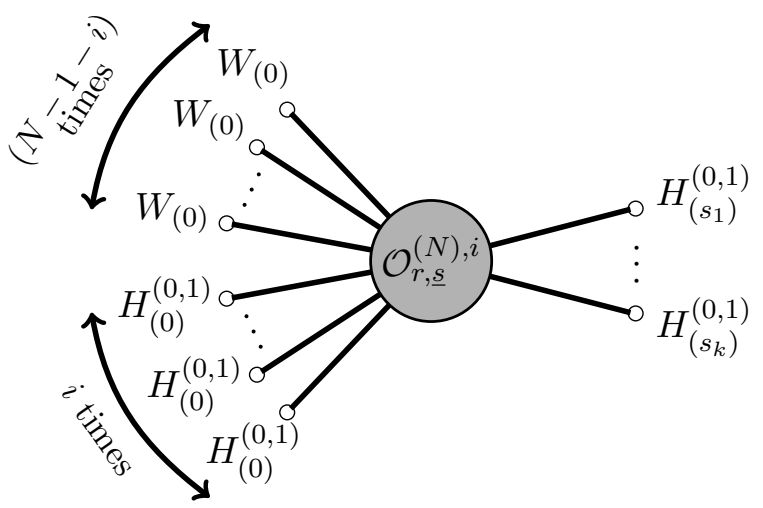

Figure 10. Schematic contribution to $B_{(s)}^{(N, r)}$ as $(N-1-i) W_{(0)}$ and $i H_{(0)}^{(0,1)}$ coupling to multiple $H_{\left(s_{a}\right)}^{(0,1)}$ through $\mathcal{O}_{r, \underline{s}}^{(N), i}$, for $i=0, \ldots, N-1$ and $a=1, \ldots, k=N(r-1)+1$.

\subsection{Modular graph functions}

Throughout the computations in sections 3 and 4 as well as in the previous subsection we have invoked a graphical representation for instanton contributions to the little string free energy that resembles higher-point functions representing effective couplings. While primarily a useful graphical device to organise the different contributions, we have already remarked previously that there might be more to it, beyond a simple graphical similarity. In this section, focusing mostly on the order $Q_{R}^{1}$, we provide further evidence to this effect.

\subsubsection{Propagator}

We start by considering the simplest (non-trivial) coupling function $\mathcal{O}^{(N), 1}$, for which we have found the expressions in eq. (3.2) for $N=2$ and eq. (4.5) for $N=3$. Following [22], these two functions can be written in terms of the Weierstrass elliptic function $\wp(z ; \rho)$ (see $($ A.8))

$$
\begin{aligned}
\mathcal{O}^{(2), 1}\left(\widehat{a}_{1}, \rho\right) & =-\frac{2}{(2 \pi i)^{2}}\left[\frac{\pi^{2}}{3} E_{2}(\rho)+\wp\left(\widehat{a}_{1} ; \rho\right)\right], \\
\mathcal{O}^{(3), 1}\left(\widehat{a}_{1}, \widehat{a}_{2}, \rho\right) & =\frac{2}{(2 \pi i)^{2}} \sum_{\ell=1}^{3}\left[\frac{\pi^{2}}{3} E_{2}(\rho)+\wp\left(\widehat{a}_{\ell} ; \rho\right)\right], \quad \text { with } \quad \widehat{a}_{3}=\rho-\widehat{a}_{1}-\widehat{a}_{2} .
\end{aligned}
$$

Written in this form, however, we can express $\mathcal{O}^{(2), 1}$ and $\mathcal{O}^{(3), 1}$ in terms of the two-point function of a free boson on the torus (see appendix A.2)

$$
\mathbb{G}(z ; \rho)=-\ln \left|\frac{\theta_{1}(z ; \rho)}{\theta_{1}^{\prime}(0, \rho)}\right|^{2}-\frac{\pi}{2 \operatorname{Im}(\rho)}(z-\bar{z})^{2} .
$$

Following $[55,56]$ the latter satisfies $\partial_{z}^{2} \mathbb{G}(z ; \rho)=\mathbb{G}^{\prime \prime}(z ; \rho)=\wp(z ; \rho)+\frac{\pi^{2}}{3} \widehat{E}_{2}(\rho)$ (where $\widehat{E}_{2}(\rho)$ is defined in (A.7)), such that we can write

$$
\begin{aligned}
\mathcal{O}^{(2), 1}\left(\widehat{a}_{1}, \rho\right) & =-\frac{2}{(2 \pi i)^{2}}\left[\mathbb{G}^{\prime \prime}\left(\widehat{a}_{1} ; \rho\right)+\frac{2 \pi i}{\rho-\bar{\rho}}\right], \\
\mathcal{O}^{(3), 1}\left(\widehat{a}_{1}, \widehat{a}_{2}, \rho\right) & =\frac{2}{(2 \pi i)^{2}} \sum_{\ell=1}^{3}\left[\mathbb{G}^{\prime \prime}\left(\widehat{a}_{\ell} ; \rho\right)+\frac{2 \pi i}{\rho-\bar{\rho}}\right], \quad \text { with } \quad \widehat{a}_{3}=\rho-\widehat{a}_{1}-\widehat{a}_{2} .
\end{aligned}
$$


This makes it clear that $\mathcal{O}^{(2), 1}\left(\widehat{a}_{1}, \rho\right)$ and $\mathcal{O}^{(3), 1}\left(\widehat{a}_{1}, \widehat{a}_{2}, \rho\right)$ are modular objects, up to a term proportional to $(\rho-\bar{\rho})^{-1}$. Since the latter is precisely the contribution to complete the $E_{2}$ in (5.4) and (5.5) into $\widehat{E}_{2}, \mathcal{O}^{(N), 1}$ are (non-holomorphic) modular objects, provided $E_{2}$ is replaced by $\widehat{E}_{2}$. A similar behaviour under modular transformations has been observed previously (see e.g. [49]) in certain expansions of the free energy.

Furthermore, using the data provided in [22], we can compute in the same manner $\mathcal{O}^{(4), 1}$ (i.e. for $N=4$ ), for which we find

$\mathcal{O}^{(4), 1}\left(\widehat{a}_{1}, \widehat{a}_{2}, \widehat{a}_{3}, \rho\right)=-\frac{2}{(2 \pi i)^{2}}\left(\frac{12 \pi i}{\rho-\bar{\rho}}+\sum_{\ell=1}^{4} \mathbb{G}^{\prime \prime}\left(\widehat{a}_{\ell} ; \rho\right)+\mathbb{G}^{\prime \prime}\left(\widehat{a}_{1}+\widehat{a}_{2} ; \rho\right)+\mathbb{G}^{\prime \prime}\left(\widehat{a}_{2}+\widehat{a}_{3} ; \rho\right)\right)$,

where $\widehat{a}_{4}=\rho-\widehat{a}_{1}-\widehat{a}_{2}-\widehat{a}_{3}$. We remark that the specific form of the last two terms in the bracket of eq. (5.8) is due to the fact that we are considering the free energy in which single-particle states have been removed. ${ }^{10}$ Including the latter in the full free energy for $N=4$ would lead to a more symmetric combination of arguments for the latter terms.

\subsubsection{Modular graph functions}

The coupling functions $\mathcal{O}^{(N), i}$ for $i>1$ are somewhat more involved. However, they still share a fair amount of properties with so-called graph functions, which have appeared in the study of Feynman diagrams in field theory as well as string theory [32, 33, 38-45]. To make contact with these recent results in the literature, we choose to represent (5.4) in a slightly different fashion [22]

$$
\mathcal{O}^{(2), 1}=-2 \mathcal{I}_{0}, \quad \text { with } \quad \mathcal{I}_{0}=\sum_{n=1}^{\infty} \frac{n}{1-Q_{\rho}^{n}}\left(Q_{\widehat{a}_{1}}^{n}+\frac{Q_{\rho}^{n}}{Q_{\widehat{a}_{1}}^{n}}\right) .
$$

The function $\mathcal{I}_{0}$ can (formally) be writen as a second derivative $\mathcal{I}_{0}=D_{\widehat{a}_{1}}^{2} \mathcal{I}_{-1}$ (where $\left.D_{\widehat{a}_{1}}=\frac{1}{2 \pi i} \frac{\partial}{\partial \widehat{a}_{1}}=Q_{\widehat{a}_{1}} \frac{\partial}{\partial Q_{\widehat{a}_{1}}}\right)$ of the function

$$
\begin{aligned}
\mathcal{I}_{-1} & =\sum_{n=1}^{\infty} \frac{n^{-1}}{1-Q_{\rho}^{n}}\left(Q_{\widehat{a}_{1}}^{n}+\frac{Q_{\rho}^{n}}{Q_{\widehat{a}_{1}}^{n}}\right)=\sum_{n=1}^{\infty} Q_{\widehat{a}_{1}}^{n}+\sum_{n=1}^{\infty} \sum_{k=1}^{\infty} k^{-1} Q_{\rho}^{n k}\left(Q_{\widehat{a}_{1}}^{k}+Q_{\widehat{a}_{1}}^{-k}\right) \\
& =\sum_{n=0}^{\infty} \operatorname{Li}_{1}\left(Q_{\rho}^{n} Q_{\widehat{a}_{1}}\right)+\sum_{n=1}^{\infty} \operatorname{Li}_{1}\left(Q_{\rho}^{n} Q_{\widehat{a}_{1}}^{-1}\right) .
\end{aligned}
$$

Comparing with the notation introduced in [33], we have

$$
2 \operatorname{Re}\left(\mathcal{I}_{-1}\right)=D_{1,1}\left(Q_{\rho}, Q_{\widehat{a}_{1}}\right)-\frac{\pi \operatorname{Im}(\rho)}{3},
$$

where we set $\zeta=Q_{\widehat{a}_{1}}$ and $u=0$. The object $D_{1,1}$ is one of the fundamental building blocks in the construction of the modular graph functions discussed in [33]. In particular, it was argued in [33] that the $I_{\alpha}:=\left(Q_{\widehat{a}_{1}} \frac{\partial}{\partial Q_{\widehat{a}_{1}}}\right)^{2 \alpha} \mathcal{I}_{0}$ (for $\alpha \in \mathbb{N}$ ), which generically appear in the

\footnotetext{
${ }^{10}$ This is due to the presence of the plethystic logarithm rather than the simple logarithm in (2.2).
} 
expansion of the free energy, are combinations of polylogarithms, thus generalising (5.10). In fact, following [22], the $\mathcal{O}^{(N), i}$ have very similar properties: they can be written as combinations of generating functions of multiple divisor sums $T\left(\widehat{a}_{1}, \ldots, \widehat{a}_{N-1} ; \rho\right)$, which have first been introduced in [26] (see appendix B for a short overview). For example, the simplest case $\mathcal{O}^{(2), 1}$ in (3.2) can be expressed as

$$
\mathcal{O}^{(2), 1}\left(\widehat{a}_{1}, \rho\right)=-2 D_{\widehat{a}_{1}}\left[T\left(\widehat{a}_{1}-\rho ; \rho\right)-T\left(-\widehat{a}_{1} ; \rho\right)\right] .
$$

In a similar fashion also other $\mathcal{O}^{(N), i}$ can be represented in terms of the generating functions of the multiple divisor sums $T\left(\widehat{a}_{1}, \ldots, \widehat{a}_{N-1} ; \rho\right)$ (see [22]). Using the definition (B.1) along with the presentation (B.4) for the latter, we see that $\mathcal{O}^{(N), i}$ can also be decomposed into polylogarithms. The main difference, however, is that the latter have in general negative level. It would nonetheless be interesting to see, if these objects still allow a presentation in terms of Eichler integrals as for example in [33]. We leave this question for future work.

\section{Conclusions}

In this paper we have continued the study of (non-perturbative) symmetries in a class of little string theories of A-type (see [21, 22]). We have focused on those theories, which in the low energy limit describe a six-dimensional gauge theory with gauge group $\mathrm{U}(N)$ (for $N \in \mathbb{N}$ ) and matter in the adjoint representation. Making use of recently discovered [21, 22] patterns in the series expansion of the free energy (which we also verified to even higher order in this work), we have organised the unrefined limit of the latter (which corresponds to the choice $\epsilon_{1}=-\epsilon_{2}$ of the deformation parameters) in a rather intriguing fashion: for $N=2,3$ and to leading order in the instanton parameter (i.e. $Q_{R}^{1}$ ), we have shown that the $B_{(s)}^{2, r}$ can be organised in a way that resembles sums of higher-point functions (almost like Feynman diagrams). Indeed, a general such contribution consists of $N$ 'external legs' out of which one is $H_{(s)}^{(0,1)}$ and the remaining $N-1$ are either $H_{(0)}^{(0,1)}$ or $W_{(0)}=\frac{1}{24}\left(\phi_{0,1}+2 E_{2} \phi_{-2,1}\right)$. These are either basic building blocks of the free energy for $N=1$ or related to the function $W$ that governs the BPS-counting of an M5-brane with a single M2-brane attached to it on either side (see [21]). These external legs are coupled through the coupling functions $\mathcal{O}^{(N), i}$ (for $i=0, \ldots, N-1$ ). The latter are functions of the roots $\widehat{a}_{1, \ldots, N-1}$ of the gauge algebra $\mathfrak{a}_{N+1}$ as well as $\rho$, but are independent of $S$ and the deformation parameter $s$ : in the simplest case, i.e. for $i=0$, the examples we have studied (including the case $N=4$ ) suggest that $\mathcal{O}^{(N), 0}=N$ is a simple constant. For $i=1, \mathcal{O}^{(N), i}$ is a non-trivial function and we have seen (for $N=2,3,4$ ) that it can be related to the second derivative of the scalar Greens function on the torus, i.e. the scalar propagator. It is this fact which leads us to believe that the diagrammatic representation is more than a mere graphical device. Indeed, higher coupling functions $\mathcal{O}^{(N), i>1}$ show certain similarities with graph functions that have been studied in the literature (see notably [33]) in connection with scattering amplitudes in string and field theory. Higher orders in the instanton parameter show similar structures, however, they are complicated by two facts: (i) these 'diagrams' contain additional legs of the form $H_{(s)}^{(0,1)}$ with more complicated coupling functions; (ii) to this order we also find 
contributions that are obtained from the leading instanton result through the action of (Hecke) operators.

The results obtained in this work lend themselves to direct generalisations in a number of directions: on the one hand side it is interesting to understand if certain similarities between the coupling functions $\mathcal{O}^{(N), i}$ and graph functions is merely a coincidence or can be made more concrete. In this regard it might be interesting whether the algebra of the generating functions of multiple divisor sums [26] leads to an algebra of the $\mathcal{O}^{(N), i}$ which is akin to recent results in the amplitude literature (see e.g. [43, 44]). On the other hand, the appearance of contributions to higher order in the instanton parameters that can be obtained through the action of (Hecke) operators on the contributions to order $Q_{R}^{1}$, is very reminiscent of the Hecke like-structures observed in [25] in a particular subsector of the free energy in the NS-limit (see also similar observations in [22]). It will be interesting in the future to see if it is possible to make this connection more concrete.

\section{Acknowledgments}

I would like to thank Brice Bastian for collaboration on the previous projects [21, 22], which have been a great influence for this work. Furthermore, I am deeply indebted to Amer Iqbal, Oliver Schlotterer and Pierre Vanhove for a careful reading of the manuscript and many useful comments and exchanges.

\section{A Modular toolbox}

In an attempt to keep this work self-contained, in this appendix we compile a minimum of relations and properties regarding modular forms, which are necessary for the discussion in the main body of this article. For more information, we refer the reader to e.g. [57].

\section{A.1 Jacobi forms and Eisenstein series}

In the main body of this paper we frequently encounter two sets of functions, namely the Jacobi forms $\phi_{-2,1}$ and $\phi_{0,1}$ as well as the Eisenstein series $E_{2 k}$. The former are defined as

$$
\phi_{0,1}(\rho, z)=8 \sum_{a=2}^{4} \frac{\theta_{a}^{2}(z ; \rho)}{\theta_{a}^{2}(0, \rho)}, \quad \text { and } \quad \phi_{-2,1}(\rho, z)=\frac{\theta_{1}^{2}(z ; \rho)}{\eta^{6}(\rho)}
$$

where $\theta_{a=1,2,3,4}(z ; \rho)$ are the Jacobi theta functions and $\eta(\rho)$ is the Dedekind eta function. These two are examples of weak Jacobi forms of index 1 and weight 0 and -2 respectively. Under a weak Jacobi form of index $m \in \mathbb{Z}$ and weight $w \in \mathbb{Z}$ for a finite index subgroup $\Gamma \subset \mathrm{SL}(2, \mathbb{Z})$, we understand a holomorphic function (here $\mathbb{H}$ is the upper complex plane)

$$
\begin{aligned}
\phi: \mathbb{H} \times \mathbb{C} & \longrightarrow \mathbb{C} \\
(\rho, z) & \longmapsto \phi(\rho ; z),
\end{aligned}
$$


with the properties

$$
\begin{aligned}
\phi\left(\frac{a \rho+b}{c \rho+d} ; \frac{z}{c \rho+d}\right) & =(c \rho+d)^{w} e^{\frac{2 \pi i m c z^{2}}{c \rho+d}} \phi(\rho ; z), & & \forall\left(\begin{array}{ll}
a & b \\
c & d
\end{array}\right) \in \Gamma, \\
\phi\left(\rho ; z+\ell_{1} \rho+\ell_{2}\right) & =e^{-2 \pi i m\left(\ell_{1}^{2} \rho+2 \ell_{1} z\right)} \phi(\rho ; z), & & \forall \ell_{1,2} \in \mathbb{N},
\end{aligned}
$$

which furthermore affords a Fourier expansion of the form

$$
\phi(z, \rho)=\sum_{n=0}^{\infty} \sum_{\ell \in \mathbb{Z}} c(n, \ell) Q_{\rho}^{n} e^{2 \pi i z \ell} .
$$

We can construct new Jacobi forms as polynomials in $\phi_{-2,1}$ and $\phi_{0,1}$ whose coefficients are given by suitable modular forms. In the case of the full modular group, the latter are generate by the ring of Eisenstein series spanned by $\left\{E_{4}, E_{6}\right\}$, where

$$
E_{2 k}(\rho)=1-\frac{4 k}{B_{2 k}} \sum_{n=1}^{\infty} \sigma_{2 k-1}(n) Q_{\rho}^{n}, \quad \forall k \in \mathbb{N},
$$

and $B_{2 k}$ are the Bernoulli numbers, while $\sigma_{k}(n)$ is the divisor sum. For certain applications, we also defined

$$
G_{2 k}(\rho)=2 \zeta(2 k)+2 \frac{(2 \pi i)^{2 k}}{(2 k-1) !} \sum_{n=1}^{\infty} \sigma_{2 k-1}(n) Q_{\rho}^{n}=2 \zeta(2 k) E_{2 k}(\rho),
$$

which differs by its normalisation. Notice that (A.5) includes the case $k=1$, where $E_{2}$ is strictly speaking not a modular form. Instead it transforms with an additional shift term under modular transformations. It can, however, be completed into $\widehat{E}_{2}$, which transforms with weight 2 under modular transformations, ate the expense of being no longer holomorphic

$$
\widehat{E}_{2}(\rho, \bar{\rho})=E_{2}(\rho)-\frac{6 i}{\pi(\rho-\bar{\rho})} .
$$

With the help of the (holomorphic) Eisenstein series we can also define the Weierstrass elliptic function

$$
\wp(z ; \rho)=\frac{1}{z^{2}}+\sum_{k=1}^{\infty}(2 k+1) G_{2 k+2}(\rho) z^{2 k} .
$$

Furthermore, we also introduce the following objects

$$
\psi_{2}(\rho)=\theta_{3}^{4}(\rho, 0)+\theta_{4}^{4}(\rho, 0)=-2\left(E_{2}(\rho)-2 E_{2}(2 \rho)\right), \quad \text { and } \quad \psi_{3}(\rho)=E_{2}-3 E_{2}(3 \rho),
$$

which appear in the free energy for $N=2$ to orders $Q_{R}^{2}$ and $Q_{R}^{3}$ respectively (see [22]). These functions are in fact (proportional to) Eisenstein series of the congruence subgroup $\Gamma_{0}(2)$ and $\Gamma_{0}(3)$, respectively (see $[58,59]$; see also [60] for a review of modular forms for congruence subgroups of $\mathrm{SL}(2, \mathbb{Z})$ of the type $\left.\Gamma_{0}(N)\right)$. 
Finally, in the main body of this work, we shall also make use of Hecke operators. The latter map Jacobi forms of index $m$ and weight $w$ into Jacobi forms of index $\mathrm{km}$ and weight $w$ for $k \in \mathbb{N}$. Specifically, let $J_{w, m}$ be the space of Jacobi forms of index $m$ and weight $w$ of $\mathrm{SL}(2, \mathbb{Z})$, then the $k$ th Hecke operator is defined as

$$
\begin{aligned}
\mathcal{H}_{k}: J_{w, m}(\Gamma) & \longrightarrow J_{w, k m}(\Gamma) \\
\phi(\rho, z) & \longmapsto \mathcal{H}_{k}(\phi(\rho, z))=k^{w-1} \sum_{\substack{d \mid k \\
b \bmod d}} d^{-w} \phi\left(\frac{k \rho+b d}{d^{2}}, \frac{k z}{d}\right) .
\end{aligned}
$$

\section{A.2 Torus propagator}

In this appendix, we provide some more background material on the scalar Greens function $\mathbb{G}(z)$, which appeared in the coupling functions $\mathcal{O}^{(N), 1}$ for $N=2,3,4$ (see (5.7) and (5.8)). Our discussion follows mainly [55, 61, 62].

Consider a free scalar field theory (with field $\phi$ ) on a torus whose periods of the two non-contractible cycles are chosen to be 1 and $\rho$ respectively. This theory is invariant under differentiable reparametrisations of the torus, local Lorentz rotations and Weyl symmetry, which gives rise to numerous Ward identitites that strongly constrain the higher-point correlation functions. The Greens function is given by the two-point function

$$
\mathbb{G}(z-w ; \rho)=\langle\phi(z) \phi(w)\rangle=-\ln \left|\frac{\theta_{1}(z-w ; \rho)}{\theta_{1}^{\prime}(0, \rho)}\right|^{2}-\frac{\pi}{2 \operatorname{Im}(\rho)}(z-w-(\bar{z}-\bar{w}))^{2} .
$$

The latter satisfies the relation (with $\Delta$ the two-dimensional Laplacian)

$$
\Delta_{z} G(z ; \rho)=4 \pi \delta^{(2)}(z)-\frac{2 \pi}{\operatorname{Im}(\rho)} .
$$

\section{B Generating functions of multiple divisor sums}

In [26] the following objects have been introduced

$$
T\left(X_{1}, \ldots, X_{\ell} ; \rho\right)=\sum_{s_{1}, \ldots, s_{\ell}>0}\left[s_{1}, \ldots, s_{N-1} ; \rho\right] X^{s_{1}-1} \ldots X^{s_{N-1}-1}
$$

which are generating functions of the following brackets of length $\ell$

$$
\left[s_{1}, \ldots, s_{\ell} ; \rho\right]=\frac{1}{\left(s_{1}-1\right) ! \ldots\left(s_{\ell}-1\right) !} \sum_{n>0} Q_{\rho}^{n} \sum_{\substack{u_{1} v_{1}+\ldots u_{\ell} v_{\ell}=n \\ u_{1}>\ldots>u_{\ell}>0}} v_{1}^{s_{1}-1} \ldots v_{\ell}^{s_{\ell}-1}
$$

These in turn generate multiple divisor sums

$$
\sigma_{r_{1}, \ldots, r_{\ell}}(n)=\sum_{\substack{u_{1} v_{1}+\ldots+u_{\ell} v_{\ell}=n \\
u_{1}>\ldots>u_{\ell}>0}} v_{1}^{r_{1}} \ldots v_{\ell}^{r_{\ell}}, \quad \text { for } \quad \begin{aligned}
& r_{1}, \ldots, r_{\ell} \in \mathbb{N} \cup\{0\} \\
& \ell, n \in \mathbb{N}
\end{aligned}
$$


which are generalisations of the usual divisor sigma $\sigma_{k}(n)$, which for example appears in the definition of the Eisenstein series (see (A.5)). The brackets of length $\ell$ can also be written in the form [26]

$$
\left[s_{1}, \ldots, s_{\ell} ; \rho\right]=\sum_{n_{1} \ldots>n_{\ell}>0} \widetilde{\operatorname{Li}}_{1-s_{1}}\left(Q_{\rho}^{n_{1}}\right) \ldots \widetilde{\mathrm{Li}}_{1-s_{\ell}}\left(Q_{\rho}^{n_{\ell}}\right)
$$

where the normalised polylogarithms

$$
\widetilde{\mathrm{Li}}_{1-s}(z)=\frac{\mathrm{Li}_{1-s}(z)}{\Gamma(s)}, \quad \text { with } \quad \mathrm{Li}_{-s}=\sum_{n>0} n^{s} z^{n}=\frac{z P_{s}(z)}{(1-z)^{s+1}} .
$$

In the last line we have assumed $|z|<1$ and we have introduced the Eulerian polynomials

$$
P_{s}(X)=\sum_{n=0}^{s-1} A_{s, n} X^{n}, \quad \text { with } \quad A_{s, n}=\sum_{i=0}^{n}(-1)^{i}\left(\begin{array}{c}
s+1 \\
i
\end{array}\right)(n+1-i)^{s} .
$$

Open Access. This article is distributed under the terms of the Creative Commons Attribution License (CC-BY 4.0), which permits any use, distribution and reproduction in any medium, provided the original author(s) and source are credited.

\section{References}

[1] S. Hohenegger, A. Iqbal and S.-J. Rey, Dual Little Strings from F-theory and Flop Transitions, JHEP 07 (2017) 112 [arXiv:1610.07916] [INSPIRE].

[2] B. Bastian, S. Hohenegger, A. Iqbal and S.-J. Rey, Dual little strings and their partition functions, Phys. Rev. D 97 (2018) 106004 [arXiv:1710.02455] [InSPIRE].

[3] B. Bastian, S. Hohenegger, A. Iqbal and S.-J. Rey, Triality in Little String Theories, Phys. Rev. D 97 (2018) 046004 [arXiv: 1711.07921] [INSPIRE].

[4] B. Bastian, S. Hohenegger, A. Iqbal and S.-J. Rey, Beyond Triality: Dual Quiver Gauge Theories and Little String Theories, JHEP 11 (2018) 016 [arXiv:1807.00186] [INSPIRE].

[5] E. Witten, Some comments on string dynamics, in Future perspectives in string theory. Proceedings, Conference, Strings'95, Los Angeles, U.S.A., 13-18 March 1995, pp. 501-523 (1995) [hep-th/9507121] [INSPIRE].

[6] P.S. Aspinwall, Point-like instantons and the $\operatorname{Spin}(32) / Z_{2}$ heterotic string, Nucl. Phys. B 496 (1997) 149 [hep-th/9612108] [INSPIRE].

[7] P.S. Aspinwall and D.R. Morrison, Point-like instantons on K3 orbifolds, Nucl. Phys. B 503 (1997) 533 [hep-th/9705104] [INSPIRE].

[8] N. Seiberg, New theories in six-dimensions and matrix description of $M$-theory on $T^{5}$ and $T^{5} / \mathbb{Z}_{2}$, Phys. Lett. B 408 (1997) 98 [hep-th/9705221] [INSPIRE].

[9] N. Kim and S.-J. Rey, M(atrix) theory on T(5)/Z(2) orbifold and five-branes, Nucl. Phys. B 534 (1998) 155 [hep-th/9705132] [inSPIRE].

[10] K.A. Intriligator, New string theories in six-dimensions via branes at orbifold singularities, Adv. Theor. Math. Phys. 1 (1998) 271 [hep-th/9708117] [INSPIRE]. 
[11] A. Hanany and A. Zaffaroni, Branes and six-dimensional supersymmetric theories, Nucl. Phys. B 529 (1998) 180 [hep-th/9712145] [INSPIRE].

[12] I. Brunner and A. Karch, Branes at orbifolds versus Hanany Witten in six-dimensions, JHEP 03 (1998) 003 [hep-th/9712143] [INSPIRE].

[13] L. Bhardwaj, M. Del Zotto, J.J. Heckman, D.R. Morrison, T. Rudelius and C. Vafa, F-theory and the Classification of Little Strings, Phys. Rev. D 93 (2016) 086002 [Erratum ibid. D 100 (2019) 029901] [arXiv: 1511.05565] [INSPIRE].

[14] L. Bhardwaj, Revisiting the classifications of 6d SCFTs and LSTs, arXiv:1903.10503 [INSPIRE].

[15] N. Haouzi and C. Kozçaz, The ABCDEFG of Little Strings, arXiv:1711.11065 [INSPIRE].

[16] S. Hohenegger, A. Iqbal and S.-J. Rey, Instanton-monopole correspondence from M-branes on $\mathbb{S}^{1}$ and little string theory, Phys. Rev. D 93 (2016) 066016 [arXiv:1511.02787] [InSPIRE].

[17] S. Hohenegger, A. Iqbal and S.-J. Rey, Self-Duality and Self-Similarity of Little String Orbifolds, Phys. Rev. D 94 (2016) 046006 [arXiv: 1605.02591] [InSPIRE].

[18] A. Kanazawa and S.-C. Lau, Local Calabi-Yau manifolds of type Ã via SYZ mirror symmetry, J. Geom. Phys. 139 (2019) 103 [arXiv:1605.00342] [INSPIRE].

[19] B. Haghighat and R. Sun, M5 branes and Theta Functions, JHEP 10 (2019) 192 [arXiv: 1811.04938] [INSPIRE].

[20] B. Bastian and S. Hohenegger, Dihedral Symmetries of Gauge Theories from Dual Calabi-Yau Threefolds, Phys. Rev. D 99 (2019) 066013 [arXiv:1811.03387] [InSPIRE].

[21] B. Bastian and S. Hohenegger, Symmetries in A-Type Little String Theories, Part I, arXiv: 1911.07276 [INSPIRE].

[22] B. Bastian and S. Hohenegger, Symmetries in A-Type Little String Theories, Part II, arXiv: 1911.07280 [INSPIRE].

[23] N.A. Nekrasov and S.L. Shatashvili, Quantization of Integrable Systems and Four Dimensional Gauge Theories, in Proceedings, 16th International Congress on Mathematical Physics (ICMP09), Prague, Czech Republic, 3-8 August 2009, pp. 265-289 (2009) [DOI] [arXiv: 0908.4052] [INSPIRE].

[24] A. Mironov and A. Morozov, Nekrasov Functions and Exact Bohr-Zommerfeld Integrals, JHEP 04 (2010) 040 [arXiv:0910.5670] [INSPIRE].

[25] A. Ahmed, S. Hohenegger, A. Iqbal and S.-J. Rey, Bound states of little strings and symmetric orbifold conformal field theories, Phys. Rev. D 96 (2017) 081901 [arXiv: 1706.04425] [INSPIRE].

[26] H. Bachmann and U. Kühn, The algebra of generating functions for multiple divisor sums and applications to multiple zeta values, Ramanujan J. (2013) [arXiv:1309.3920] [INSPIRE].

[27] O. Schlotterer and S. Stieberger, Motivic Multiple Zeta Values and Superstring Amplitudes, J. Phys. A 46 (2013) 475401 [arXiv:1205.1516] [inSPIRE].

[28] J. Broedel, O. Schlotterer, S. Stieberger and T. Terasoma, All order $\alpha^{\prime}$-expansion of superstring trees from the Drinfeld associator, Phys. Rev. D 89 (2014) 066014 [arXiv: 1304.7304] [INSPIRE].

[29] J. Broedel, O. Schlotterer and S. Stieberger, Polylogarithms, Multiple Zeta Values and Superstring Amplitudes, Fortsch. Phys. 61 (2013) 812 [arXiv:1304.7267] [INSPIRE]. 
[30] S. Stieberger, Closed superstring amplitudes, single-valued multiple zeta values and the Deligne associator, J. Phys. A 47 (2014) 155401 [arXiv:1310.3259] [INSPIRE].

[31] J. Broedel, C.R. Mafra, N. Matthes and O. Schlotterer, Elliptic multiple zeta values and one-loop superstring amplitudes, JHEP 07 (2015) 112 [arXiv:1412.5535] [INSPIRE].

[32] J. Broedel, N. Matthes and O. Schlotterer, Relations between elliptic multiple zeta values and a special derivation algebra, J. Phys. A 49 (2016) 155203 [arXiv: 1507.02254] [INSPIRE].

[33] E. D'Hoker, M.B. Green, Ö. Gürdogan and P. Vanhove, Modular Graph Functions, Commun. Num. Theor. Phys. 11 (2017) 165 [arXiv:1512.06779] [INSPIRE].

[34] N. Matthes, Elliptic multiple zeta value, Ph.D. Thesis, Universität Hamburg, (2016).

[35] F. Brown, A class of non-holomorphic modular forms I, Res. Math. Sci. 5 (2018) 7 [arXiv: 1707.01230] [INSPIRE].

[36] F. Brown, A class of non-holomorphic modular forms II: equivariant iterated Eisenstein integrals, arXiv:1708.03354.

[37] J. Broedel, O. Schlotterer and F. Zerbini, From elliptic multiple zeta values to modular graph functions: open and closed strings at one loop, JHEP 01 (2019) 155 [arXiv:1803.00527] [INSPIRE].

[38] F. Zerbini, Elliptic multiple zeta values, modular graph functions and genus 1 superstring scattering amplitudes, Ph.D. Thesis, Bonn U. (2017) [arXiv:1804.07989] [INSPIRE].

[39] E. D'Hoker and M.B. Green, Identities between Modular Graph Forms, J. Number Theor. 189 (2018) 25 [arXiv: 1603.00839] [INSPIRE].

[40] E. D'Hoker, M.B. Green and B. Pioline, Higher genus modular graph functions, string invariants and their exact asymptotics, Commun. Math. Phys. 366 (2019) 927 [arXiv: 1712.06135] [INSPIRE].

[41] F. Zerbini, Modular and Holomorphic Graph Functions from Superstring Amplitudes, in Proceedings, KMPB Conference: Elliptic Integrals, Elliptic Functions and Modular Forms in Quantum Field Theory, Zeuthen, Germany, 23-26 October 2017, pp. 459-484 (2019) [DOI] [arXiv: 1807.04506] [INSPIRE].

[42] J.E. Gerken, A. Kleinschmidt and O. Schlotterer, Heterotic-string amplitudes at one loop: modular graph forms and relations to open strings, JHEP 01 (2019) 052 [arXiv:1811.02548] [INSPIRE].

[43] C.R. Mafra and O. Schlotterer, All-order $\alpha^{\prime}$-expansion of one-loop open-string integrals, arXiv: 1908.09848 [INSPIRE].

[44] C.R. Mafra and O. Schlotterer, One-loop open-string integrals from differential equations: all-order $\alpha^{\prime}$-expansions at $n$ points, arXiv:1908.10830 [INSPIRE].

[45] J.E. Gerken, A. Kleinschmidt and O. Schlotterer, All-order differential equations for one-loop closed-string integrals and modular graph forms, JHEP 01 (2020) 064 [arXiv:1911.03476] [INSPIRE].

[46] B. Haghighat, A. Iqbal, C. Kozçaz, G. Lockhart and C. Vafa, M-Strings, Commun. Math. Phys. 334 (2015) 779 [arXiv: 1305.6322] [InSPIRE].

[47] B. Haghighat, C. Kozcaz, G. Lockhart and C. Vafa, Orbifolds of M-strings, Phys. Rev. D 89 (2014) 046003 [arXiv:1310.1185] [INSPIRE]. 
[48] S. Hohenegger and A. Iqbal, $M$-strings, elliptic genera and $\mathcal{N}=4$ string amplitudes, Fortsch. Phys. 62 (2014) 155 [arXiv:1310.1325] [INSPIRE].

[49] S. Hohenegger, A. Iqbal and S.-J. Rey, M-strings, monopole strings and modular forms, Phys. Rev. D 92 (2015) 066005 [arXiv:1503.06983] [InSPIRE].

[50] M. Aganagic, A. Klemm, M. Mariño and C. Vafa, The Topological vertex, Commun. Math. Phys. 254 (2005) 425 [hep-th/0305132] [INSPIRE].

[51] T.J. Hollowood, A. Iqbal and C. Vafa, Matrix models, geometric engineering and elliptic genera, JHEP 03 (2008) 069 [hep-th/0310272] [INSPIRE].

[52] A. Iqbal, C. Kozcaz and C. Vafa, The Refined topological vertex, JHEP 10 (2009) 069 [hep-th/0701156] [INSPIRE].

[53] A. Weil, Elliptic Functions according to Eisenstein and Kronecker, Springer-Verlag (1976).

[54] A. Ahmed, S. Hohenegger and A. Iqbal, work in progress.

[55] T. Eguchi and H. Ooguri, Conformal and Current Algebras on General Riemann Surface, Nucl. Phys. B 282 (1987) 308 [INSPIRE].

[56] S.M. Kuzenko and O.A. Solovev, Equations for two point correlation functions on compact Riemann surfaces, Theor. Math. Phys. 88 (1991) 901 [INSPIRE].

[57] M. Eichler and D. Zagier, The Theory of Jacobi Forms, Springer Verlag (1985).

[58] S. Lang, Introduction to Modular Forms, Grundlehren der Mathematischen Wissenschaften 222, Springer Verlag, Berlin (1995).

[59] W. Stein, Modular Forms, a Computational Approach, Graduate Studies in Mathematics 79, American Mathematical Society, Providence, RI (2007).

[60] M.R. Gaberdiel, S. Hohenegger and R. Volpato, Mathieu Moonshine in the elliptic genus of K3, JHEP 10 (2010) 062 [arXiv: 1008.3778] [INSPIRE].

[61] J. Polchinski, String theory. Vol. 1: An introduction to the bosonic string, Cambridge University Press, (2007) [INSPIRE].

[62] E. D'Hoker and D.H. Phong, The Geometry of String Perturbation Theory, Rev. Mod. Phys. 60 (1988) 917 [INSPIRE]. 